\title{
A Self-standing Organic Supercapacitor to Power Bioelectronic Devices
}

\author{
Georgios Nikiforidis, Shofarul Wustoni, David Ohayon, Victor Druet, and Sahika Inal* \\ Cite This: ACS Appl. Energy Mater. 2020, 3, 7896-7907 \\ Read Online
}

ABSTRACT: The last decade has witnessed rapid progress in the development of implantable and wearable bio(chemical) sensors, which allow for real-time, continuous health monitoring. Among different device configurations, organic electrochemical transistors (OECTs) have shown great potential in transducing weak biological signals with on-site amplification and as components of complex circuits with low power requirements. Yet, a significant technological challenge remains in the way these devices are integrated with power sources that are conventionally bulky and rigid. Here, we present a simple process to assemble a supercapacitor (SC) that is selfstanding, lightweight, and biocompatible and made of two identical conducting polymer (poly(3,4-ethylenedioxythiophene) electrodes

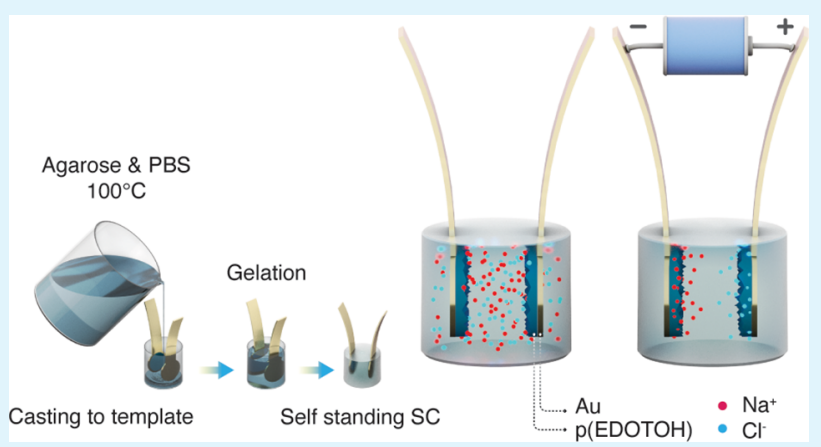
and an agarose hydrogel comprising alkali metal halides. This SC is distinguished by its high energy and power density $\left(20 \mathrm{Wh} \mathrm{kg}^{-1}\right.$ and $10^{5} \mathrm{~W} \mathrm{~kg}^{-1}$, respectively), moderate gravimetric specific capacitance $\left(70 \mathrm{~F} \mathrm{~g}^{-1}\right)$, excellent stability (charge retention of $75 \%$ after 12,000 cycles), operational flexibility (can accommodate various types of aqueous electrolytes), long-lasting self-discharge ( $>10 \mathrm{~h}$ ), and fast response time (between 0.1 and $30 \mathrm{~s}$ ). We use the SC to power a micron-scale OECT, which selectively detects sodium ions in aqueous media. When miniaturized, the SC maintains its high performance and delivers a volumetric capacitance of $240 \mathrm{~F} \mathrm{~cm}^{-3}$, highlighting the possibility of fabrication in nonstandard form factors to couple with various bioelectronic devices. This low-cost and portable power source instigates the development of robust and biocompatible onboard power sources to be implemented alongside biosensors.

KEYWORDS: organic Supercapacitor, electropolymerization, PEDOT, agarose hydrogel, electrochemical transistor, bioelectronics

\section{INTRODUCTION}

Bioelectronic devices electronically communicate with living systems to detect or control biological events. ${ }^{1}$ This communication led to the development of wearable and implantable devices used for personalized health monitoring and precision therapies. ${ }^{2}$ Key to these technologies are functional electronic materials such as conjugated polymers that combine a set of features including mixed electronic and ionic conductivity, softness, mechanical flexibility, and biocompatibility, alongside synthetic diversity and tunability., These organic mixed conductors store and transport ionic charges in the bulk of their films upon application of an electrical field through an electrolyte. The voltage-dependent charging (i.e., redox or doping state) of conjugated polymer films has been exploited in the organic electrochemical transistor (OECT) configuration, where the conjugated polymer constitutes the channel in contact with the electrolyte. As a result of the volumetric charging of the channel material gated with an electrolyte solution, OECTs are identified with large changes in the channel current for small gate voltage modulations (i.e., high transconductance, $\left.g_{\mathrm{m}}\right)^{5}$ and operate within a narrow voltage window (up to $\pm 1 \mathrm{~V}$ vs $\mathrm{Ag} \mid \mathrm{AgCl}$ ). ${ }^{6}$
Because of these features, OECTs are ideal for biosensing applications, where the device converts ionic biological signals into an electronic output while amplifying the input signals. ${ }^{7}$ OECTs have thus been used to record physiological signals when interfacing electrically active tissues, ${ }^{8}$ detect hormones from the sweat when interfacing the skin, ${ }^{9}$ sense minute concentrations of metabolites, ${ }^{10}$ ions, ${ }^{11,12}$ and proteins, ${ }^{13}$ and monitor the integrity of barrier-forming cells. ${ }^{14}$

Such sensors show great potential at the biological interface, yet one aspect that remains inadequately addressed is the compatibility of their power sources. Power devices that are coupled with biosensors should be portable, low-weight, onchip integrated, and compatible with the biological environment that the sensor is exposed to. The device components should be low cost and easy to produce and assemble. In this

Received: June 5, 2020

Accepted: July 27, 2020

Published: July 27, 2020 
Scheme 1. Formation, Components, and Operation of the Self-standing Organic Supercapacitor (for Simplicity, Only $\mathrm{Na}^{+}$and $\mathrm{Cl}^{-}$of the Buffer Are Shown as the Ions Entrapped in the Gel)

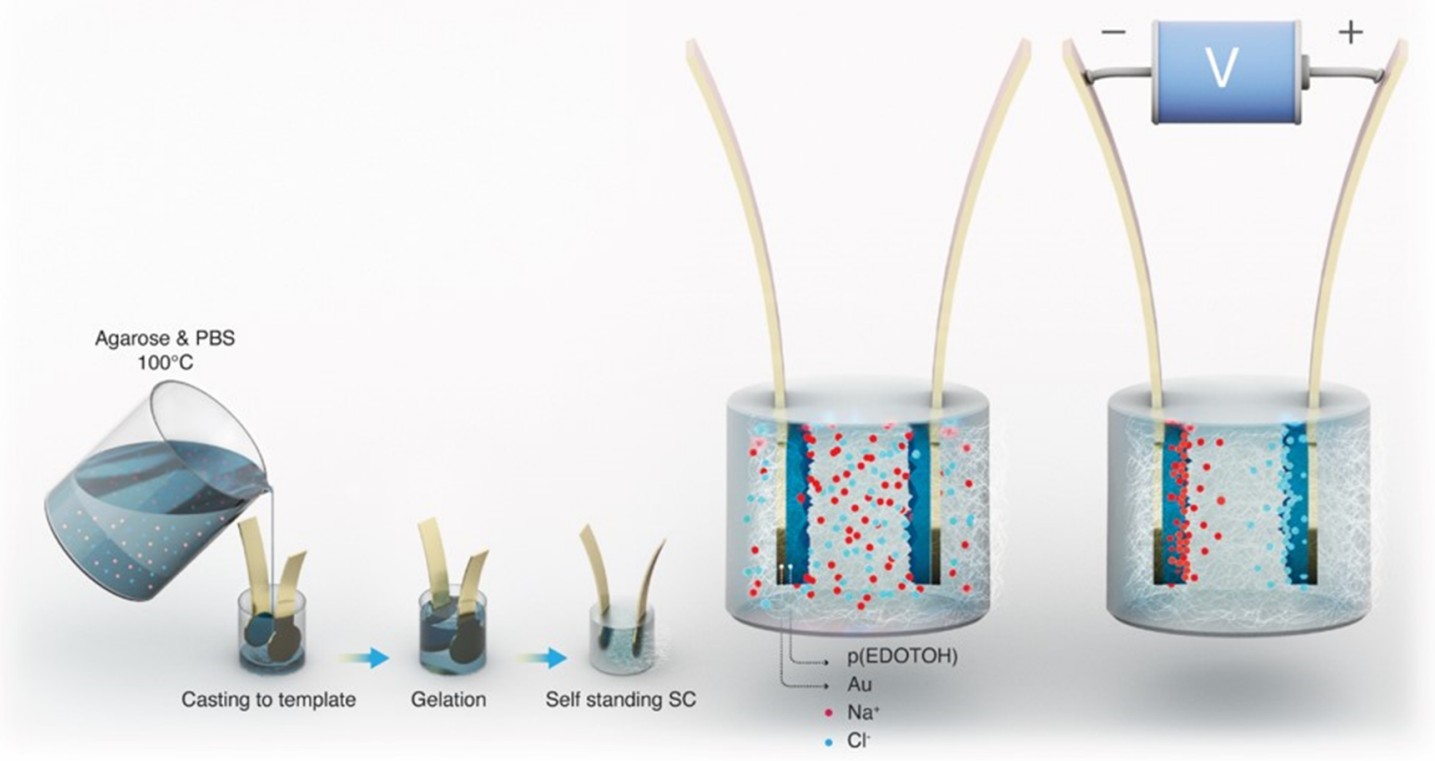

sense, designing long-lasting and sustainable powering devices should go hand in hand with the advances in sensor development. ${ }^{15,16}$ With their capability for on-demand power supply, supercapacitors (SCs) fulfill the criteria set for a powering device. ${ }^{17}$ In an SC, electrical power is directly stored as electrostatic power without any energy conversion. ${ }^{18}$ As such, they are considered as power sources, with a power density between 5 and $15 \mathrm{~kW} \mathrm{~kg}^{-1}$ (as compared to $150 \mathrm{~W}$ $\mathrm{kg}^{-1}$ for batteries $\left.{ }^{19}\right)$. While the specific energy is rather low (5-20 $\left.\mathrm{Wh} \mathrm{kg}^{-1}\right)$, SCs show a fast charge/discharge rate and long operation life, ${ }^{20}$ ideal to switch ON/OFF or monitor the output of a bioelectronic device (e.g., an OECT, identified with low power, $<1 \mu \mathrm{W}$, requirements). Depending on their charge storage mechanism, SCs are classified as electrochemical double-layer capacitors (EDLCs), pseudocapacitors (PDs), and hybrid capacitors. EDLCs typically use high surface area carbon electrodes, which store/deliver charge by an electrostatic process. ${ }^{21}$ PDs are made of conducting polymers and transition metallic oxides that are charged/discharged by fast and reversible redox processes. ${ }^{20,22}$ Hybrid capacitors consist of an EDLC electrode and a PD or battery-type electrode, combining the properties of both systems.

While carbon-based SCs hold the lion's share in SC development, large-area electrode production from highly graphitized carbon nanotubes or graphene remains an issue due to difficulties associated with processing in the solution phase. Just as for the field of bioelectronics, conducting polymers are a promising active material to build SCs owing to their ease of processability, softness, flexibility, high pseudocapacitance, and conductivity. ${ }^{23}$ In particular, poly(ethylenedioxythiophene) (PEDOT) derivatives are uniquely suited for SC development due to their ability to undergo fast and reversible ion uptake and efficient ion-electron coupling. ${ }^{24}$ They have a high theoretical conductivity $\left(>500 \mathrm{~S} \mathrm{~cm}^{-1}\right),{ }^{25}$ moderate gravimetric specific capacitance $\left(210 \mathrm{~F} \mathrm{~g}^{-1}\right),{ }^{26}$ high chemical and physical stability, and a wide operational potential window. PEDOT-based capacitors are typically made of PEDOT films that are nanostructured to achieve large surface areas. These PEDOT electrodes are, for instance, in the form of nanotubes polymerized in a porous alumina membrane yielding a specific capacitance of $132 \mathrm{~F} \mathrm{~g}^{-127}$ or fibrous films electrodeposited on graphite foil $\left(184.5 \mathrm{~F} \mathrm{~g}^{-1}\right){ }^{28}$ porous carbon paper $\left(154 \mathrm{~F} \mathrm{~g}^{-1}\right),{ }^{29}$ or cellulose paper $(115 \mathrm{~F}$ $\left.\mathrm{g}^{-1}\right) .{ }^{30}$ Nanofibers were also vapor deposited on a carbon fiber paper $\left(175 \mathrm{~F} \mathrm{~g}^{-1}\right)^{31}$ or integrated with flexible 3D carbon fiber cloths $\left(198 \mathrm{~F} \mathrm{~g}^{-1}\right)$. $^{32}$ Some other reports developed highcapacitance composites comprising PEDOT. Such SC electrodes involve PEDOT films mixed with cellulose nanofibrils and sulfonated lignin $\left(230 \mathrm{~F} \mathrm{~g}^{-1}\right){ }^{33}$ hydrogels with polyaniline $\left(112.6 \mathrm{~F} \mathrm{~g} \mathrm{~g}^{-1}\right),{ }^{34}$ and composites with graphene $(270 \mathrm{~F}$ $\left.\mathrm{g}^{-1}\right),{ }^{35,36}$ and $\mathrm{MnO}_{2}\left(1068 \mathrm{~F} \mathrm{~g}^{-1}\right){ }^{37}$ In addition, freestanding PEDOT materials have been fabricated through various polymerization methods, including an electrochemical rustvapor phase and in situ chemical polymerization, in the form of nanofibrillar films, ${ }^{31,38}$ solid-state nanowire flexible films, ${ }^{39}$ and scaffolds, ${ }^{40}$ all realized into SC devices. The specific capacitance of these SCs varies between 25 and $181 \mathrm{~F} \mathrm{~g}^{-1}$, depending on the fabrication procedure and physicochemical characteristics of the freestanding substrate. ${ }^{41-43}$ Apart from its use in SCs, PEDOT has also been the building block of the majority of OECT-based sensors (used in the channel and/or as the gate electrode coating) due to its high electronic and ionic mobilities $\left(\sim 1\right.$ to 2 and $1 \times 10^{-3} \mathrm{~cm}^{2} \mathrm{~V}^{-1} \mathrm{~s}^{-1}$, respectively $)^{44}$ and electrochemical stability.

A number of ways to prepare the PEDOT films have been reported in the literature such as chemical polymerization, electropolymerization, vapor-phase polymerization, and thermal polymerization. ${ }^{45}$ of these techniques, the electropolymerization of the corresponding monomer, EDOT, embodies a straightforward, robust, and cost-effective route to realize high-quality films on any conducting substrate. This technique combines the polymerization and geometrical patterning steps into one process while providing control over film topography and thickness through several process parameters such as applied deposition charge, counterion concentration, solvent, and electrodeposition method. ${ }^{46,47}$ In this work, we developed a self-standing organic SC comprising electropolymerized conducting polymer electrodes to enable 
high power energy storage for bioelectronic devices (Scheme 1). The SC has two components: an electropolymerized hydroxymethyl PEDOT, namely, $\mathrm{p}(\mathrm{EDOTOH})$, and an agarose hydrogel hosting hydrated ions. The in situ gelation of agarose resulted in a semiflexible, self-standing, water-filled gel with a porous structure, embedded with $\mathrm{p}(\mathrm{EDOTOH})$ electrodes. The agarose hydrogel thus fulfilled two tasks in one: it acted as an ion reservoir for the SC electrodes and provided mechanical support to the system. We analyzed the electrochemical properties of SCs built with different concentrations of hydrogels and the chemical composition of the components before and after excessive charge-discharge cycles to engineer an SC with optimal geometry and stability. Our biocompatible SC showed elaborate power density $(20 \mathrm{~W}$ $\left.\mathrm{kg}^{-1}\right)$, long-lasting self-discharge $(>10 \mathrm{~h})$, and fast response time (between 0.1 and $30 \mathrm{~s}$ ). We showed that the SC was lightweight $(0.7 \mathrm{~g})$ and robust with a capacitance retention of $75 \%$ after 12,000 cycles and that it can also be miniaturized. When integrated with microscale OECTs, the SC provided enough power to operate the device that detects variations in electrolyte concentration in real time. This is the first demonstration of electropolymerized PEDOT films in a selfstanding SC comprising agarose. We describe a unique, facile, and fast assembly of the device in a stable electrolyte-bearing hydrogel environment and present how this device can be integrated as a power source for small-power bioelectronics. Focusing on the optimization of an SC for bioelectronic device coupling, this study follows the paradigm of developing green, safe (nontoxic, nonflammable, and no-leakage), and low-cost SCs for low-power bioelectronics.

\section{EXPERIMENTAL SECTION}

2.1. Materials. Hydroxymethyl-3,4-ethylenedioxythiophene (EDOTOH), sodium chloride $(\mathrm{NaCl})$, potassium chloride $(\mathrm{KCl})$, sodium nitrate $\left(\mathrm{NaNO}_{3}\right)$, lithium perchlorate $\left(\mathrm{LiClO}_{4}\right)$, sulfuric acid (98\%, $\mathrm{H}_{2} \mathrm{SO}_{4}$ ), (3-glycidyloxypropyl)trimethoxysilane (GOPS), sodium dodecylbenzenesulfonate (DBSA), ethylene glycol (EG), and phosphate-buffered saline $(1 \times \mathrm{PBS}, \mathrm{pH}=7.4)$, and agarose $\left(M_{\mathrm{W}}=\right.$ $120,000)$ were purchased from Sigma-Aldrich and used as received. The channel of OECTs is composed of PEDOT:PSS (PH1000, Heraeus). The proton exchange membranes investigated include Nafion 117 (183 $\mu \mathrm{m}$ thickness, Sigma Aldrich) and cellulose (90 $\mu \mathrm{m}$ thickness, GoodFellow Cambridge Limited). The aqueous solutions were prepared using ultrapure water (Millipore Milli-Q).

2.2. Electrodeposition of $\mathrm{p}(\mathrm{EDOTOH})$ Films. PEDOT films were deposited on $175 \mu$ m-thick flexible Kapton (polyimide) substrates. The substrate was cut with a laser into a specific circular geometry (either 2.8 or $8 \mathrm{~mm}$ in diameter) and subsequently washed in acetone/isopropyl alcohol and deionized (DI) water. We then sputtered a 10/100 nm-thick $\mathrm{Cr} / \mathrm{Au}$ layer on the substrate followed by cleaning in acetone and soaking in DI water under sonication for $30 \mathrm{~min}$. Prior to electrodeposition, these substrates were electrochemically cleaned in an aqueous solution of $\mathrm{H}_{2} \mathrm{SO}_{4}(10 \mathrm{mM})$ using cyclic voltammetry (CV) between -0.2 and $1.2 \mathrm{~V}$ for 20 cycles (Autolab PGSTAT128N, MetroOhm). Three-electrode electrodeposition was performed by using a Pt wire as the counter electrode and a $\mathrm{Ag} \mid \mathrm{AgCl}$ reference electrode $\left(3 \mathrm{M} \mathrm{KCl}, E^{0}=0.22 \mathrm{~V}\right.$ vs standard hydrogen electrode at $\left.25{ }^{\circ} \mathrm{C}\right)$. The $\mathrm{p}(\mathrm{EDOTOH})$ film was electropolymerized in an aqueous solution containing $10 \mathrm{mM}$ EDOTOH and $100 \mathrm{mM} \mathrm{LiClO}_{4}$ using the potentiostatic mode of the potentiostat (Autolab PGSTAT128N, MetroOhm) at $1 \mathrm{~V}$ for 60 , 120,300 , and $600 \mathrm{~s}$. The synthesized films were rinsed with DI water, sprayed with $\mathrm{N}_{2}$ to remove any material weakly bound to the surface, and dried at room temperature for $24 \mathrm{~h}$. The mass of the $\mathrm{p}(\mathrm{EDOTOH})$ films was determined as the weight difference between coated and uncoated substrates using a microbalance (XP2U, Mettler Toledo).

2.3. Preparation of Agarose Gel and Assembling the Selfstanding SC. To prepare the agarose gel, a set amount of lowmelting-point agarose powder was introduced into $10 \mathrm{~mL}$ of PBS, and the solution was vigorously stirred on a hot plate set at $100{ }^{\circ} \mathrm{C}$. For instance, $1 \mathrm{wt} \%$ hydrogel corresponds to $0.1 \mathrm{~g}$ of agarose in $10 \mathrm{~mL}$ in PBS. Once the agarose was completely dissolved, the solution was cast onto the template with $\mathrm{p}(\mathrm{EDOTOH})$ electrodes facing each other at a predetermined distance, and the gel was left to cure at room temperature for $15 \mathrm{~min}$. The PBS-containing agarose gel with embedded $\mathrm{p}(\mathrm{EDOTOH})$ electrodes constitutes the self-standing allpolymer hydrogel SC (Scheme 1). The gelation results in the formation of a semiflexible water-filled gel with a $3 \mathrm{D}$ porous structure. $^{48}$ Due to the high water content $(>90 \%)$ and interconnected pore structure, the agarose hydrogel is a soft, moldable, and biocompatible medium with high ion mobility. ${ }^{49}$ This device was wetted every $4 \mathrm{~h}$, with $0.2 \mathrm{~mL}$ of $0.1 \mathrm{M} \mathrm{NaCl}$ to avoid drying and weight loss. For the case of the H-cell, the distance between the anode and the cathode was $\sim 2 \mathrm{~cm}$. The custom-built cell containing the membrane was made from poly(methyl methacrylate) and could accommodate $2.5 \mathrm{~mL}$ of the solution on each side.

2.4. Electrochemical Characterization and Performance Evaluation of the SC. Three-electrode experiments were performed in $0.1 \mathrm{M} \mathrm{NaCl}$ using a Pt wire as the counter electrode and a $\mathrm{Ag} / \mathrm{AgCl}$ reference electrode $\left(3 \mathrm{M} \mathrm{KCl}, E^{0}=0.22 \mathrm{~V}\right.$ vs standard hydrogen electrode at $25{ }^{\circ} \mathrm{C}$ ). The electrochemical properties and stability of the $\mathrm{p}(\mathrm{EDOTOH})$ film were determined by $\mathrm{CV}$ and galvanostatic charge-discharge cycling (GCD). CV and GCD curves at different current densities (i.e., charge and discharge rates, which are specified in units of current per electrode mass) were acquired in the potential window between -0.5 and $0.7 \mathrm{~V}$ at room temperature (VSP-300, BioLogic, Science Instruments). To determine the double-layer capacitance of the electrode $\left(C_{\mathrm{d}}, \mathrm{F}\right)$, the current density $(j, \mathrm{~mA}$ $\left.\mathrm{cm}^{-2}\right)$ in the non-Faradaic region $(0.3 \mathrm{~V}$ vs $\mathrm{Ag} / \mathrm{AgCl})$ of an average of three $\mathrm{CV}$ curves at different scan rates $\left(5\right.$ to $100 \mathrm{mV} \mathrm{s}^{-1}$ ) was selected. $C_{\mathrm{dl}}$ was then determined by plotting the difference in the anodic and cathodic current density $\left(\Delta j=j_{\mathrm{a}}-j_{\mathrm{c}}\right)$ against the scan rate $(\nu)$ according to the following equation:

$$
C_{\mathrm{dl}}=\frac{\mathrm{d}(\Delta j)}{2 \times \mathrm{d} \nu}=\frac{\mathrm{d}\left(j_{\mathrm{a}}-j_{\mathrm{c}}\right)}{2 \times \mathrm{d} \nu}
$$

For the SC, the specific capacitance $\left(\mathrm{F} \mathrm{g}^{-1}\right)$, which is the capacitance of one electrode per unit mass for one electrode, was determined from the GCD plots using the following equation ${ }^{50}$

$$
\text { specific capacitance }=\frac{4 \times C}{m}=\frac{4 \times I}{m \times \frac{\mathrm{d} V}{\mathrm{~d} t}}
$$

where $I$ is the discharge current applied, $m$ is the mass of the active material, and $\mathrm{d} V / \mathrm{d} t$ stems from the slope of the discharge curve, equal to $\left(V_{\max }-1 / 2 V_{\max }\right) /\left(t_{2}-t_{1}\right)$, where $V_{\max }$ corresponds to the highest voltage in the GCD curve after the voltage drop at the beginning of the discharging process. The multiplier of 4 adjusts the capacitance of two-electrode cells to the capacitance of a single electrode. The volumetric capacitance was calculated according to the following equation $^{51}$

volumetric capacitance $=\rho \times$ specific capactiance

where $\rho$ is the apparent density of the films, given by

$$
\rho=\frac{m}{A \times d}
$$

where $m$ is the mass of the dry electrode and $A\left(\mathrm{~cm}^{2}\right)$ and $d(\mathrm{~cm})$ are the area and thickness of the film electrode, respectively. The Coulombic efficiency $\left(n_{\text {Coulombic }}\right)$ of the SC was evaluated as the ratio between the discharging and charging times, $t_{\mathrm{d}}$ and $t_{\mathrm{c}}$, respectively, for the electrochemical window between $0 \mathrm{~V}$ and $0.8 \mathrm{~V}$ as displayed below 52 
(a)

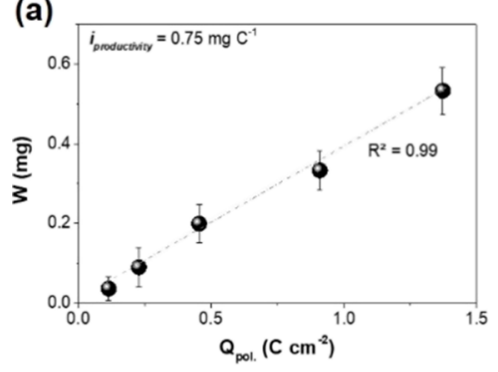

(b)

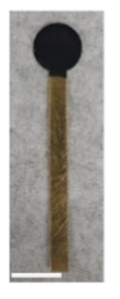

(c)

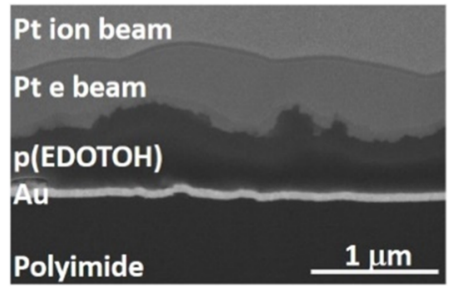

(d)

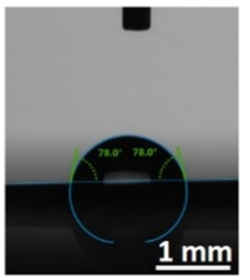

(e)

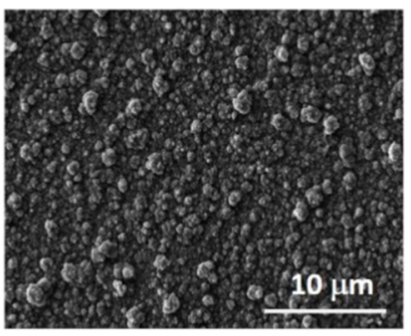

(f)

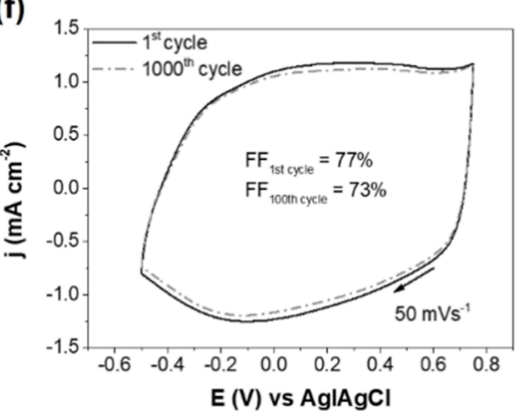

(g)

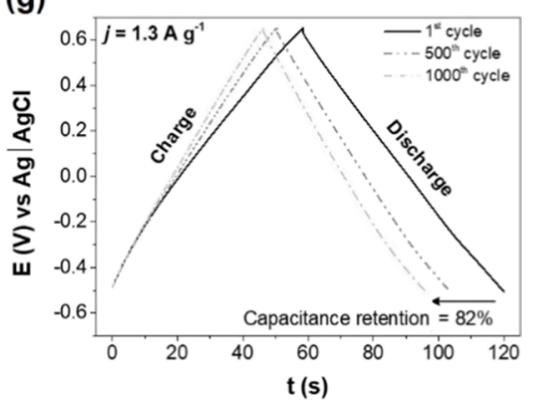

Figure 1. Electrochemical and physicochemical characterization of the $\mathrm{p}(\mathrm{EDOTOH})$ film. (a) Weight $(W)$ of $\mathrm{p}(\mathrm{EDOTOH})$ films (deposited on polyimide electrodes from an aqueous solution of $10 \mathrm{mM} \mathrm{EDOTOH}$ and $\left.100 \mathrm{mM} \mathrm{LiClO}_{4}\right)$ against the polymerization charge consumed $\left(Q_{\mathrm{pol}}\right)$. Triplicate experiments were conducted for each sample. (b) Photograph, (c) FIB-SEM, (d) water contact angle, and (e) top-view SEM image of a $\mathrm{p}(\mathrm{EDOTOH})$ film electropolymerized on a Au-coated polyimide substrate. (f) Repetitive cyclic voltammetry (CV) at $50 \mathrm{mV} \mathrm{s}$ in $0.1 \mathrm{M} \mathrm{NaCl}$ at $25{ }^{\circ} \mathrm{C}$ and (g) 1000 galvanostatic charge-discharge (GCD) cycles recorded at a normalized current density of $1.3 \mathrm{~A} \mathrm{~g}^{-1}$.

$$
n_{\text {Coulombic }}=\frac{\text { total charge released }}{\text { total charge stored }}=\frac{\text { discharging time }}{\text { charging time }}=\frac{t_{\mathrm{d}}}{t_{\mathrm{c}}}
$$

The cycling stability of the devices was studied by applying charge-discharge cycles at different current densities (i.e., 3.44 and $6.89 \mathrm{~A} \mathrm{~g}^{-1}$ ) in a two-electrode configuration from $0 \mathrm{~V}$ to $0.8 \mathrm{~V}$, which corresponds to $t_{\mathrm{c}}$ and $t_{\mathrm{d}}$ of approximately 5 to $20 \mathrm{~s}$. The energy density $\left(E, \mathrm{Wh} \mathrm{kg}^{-1}\right)$, which is the amount of energy stored per unit of mass, and the power density $\left(P, \mathrm{~W} \mathrm{~kg}^{-1}\right)$, which describes the speed at which the energy stored can be delivered, were derived from the GCD curves by applying the following equations: ${ }^{50,52,53}$

$$
\begin{aligned}
& E=\frac{1}{2} \times V_{\max }^{2} \times \text { specific capacitance } \\
& P=\frac{E}{t_{\mathrm{d}}}
\end{aligned}
$$

The self-discharge of the SC was monitored by applying $0.4 \mathrm{~mA}$ (i.e., $6.89 \mathrm{~A} \mathrm{~g}^{-1}$ for 5-30 s) followed by an open-circuit potential recording. An SC cell was assembled and charged for $30 \mathrm{~s}$ to drive a red $(1.35 \mathrm{~V})$ light-emitting diode (LED).

Electrochemical impedance spectroscopy (EIS) for the SC was performed at the open-circuit potential using a VSP-300 BioLogic potentiostat. The AC amplitude was $10 \mathrm{mV}$, while the frequencies varied between $0.01 \mathrm{~Hz}$ and $100 \mathrm{kHz}$. The open-circuit voltage (OCV) of the SC was monitored by the VSP-300 BioLogic potentiostat. The leakage current $\left(J_{\text {leak }}\right)$ of the SC was recorded using an electrical instrument analyzer (Keithley 2002, Multimeter) controlled by customized LabVIEW software.

2.5. Physicochemical Characterization. The surface morphology of the electrodeposited $\mathrm{p}(\mathrm{EDOTOH})$ film was characterized using an FEI Nova nanoscanning electron microscope (SEM) with an accelerating voltage of $3 \mathrm{kV}$ and a working distance of $5 \mathrm{~mm}$ and an atomic force microscope (AFM). The films were mounted onto aluminum stubs and attached with conductive tape. The crosssectional image was examined with a focused ion beam (FIB)/SEM (FEI Helios NanoLab 400S) using a $\mathrm{Ga}^{+}$ion source. Platinum layers were deposited on the surface region of interest by electron and ion beams for sample protection. AFM images were obtained with a Veeco Dimension 3100 scanning probe system. The images of the dried samples were obtained in tapping mode in air using FESPA-V2 probes commercialized by Bruker (nominal resonant frequency: 75 $\mathrm{KHz}$, spring constant: $2.8 \mathrm{~N} \cdot \mathrm{m}^{-1}$ ). Morphological images in a liquid environment obtained with a Bruker Scanasyst-fluid module and corresponding Scanasyst-fluid probes (nominal resonant frequency: $150 \mathrm{KHz}$, spring constant: $0.7 \mathrm{~N} \cdot \mathrm{m}^{-1}$ ). Statistical data were collected on the surface using the Gwyddion software. The chemical composition of the $\mathrm{p}(\mathrm{EDOTOH})$ before and after the electrochemical measurements was characterized by X-ray photoelectron spectroscopy (XPS), Fourier transform infrared (FTIR), and ultraviolet-visible spectroscopy (UV-vis). XPS was carried out using a KRATOS Analytical AMICUS instrument equipped with an $\mathrm{Al} \mathrm{K} \alpha \mathrm{X}$-ray source $(1468.6 \mathrm{eV})$. The source operated at a voltage of $10 \mathrm{kV}$ and a current of $10 \mathrm{~mA}$ generating a power of $100 \mathrm{~W}$. The high-resolution XPS spectra were acquired with a step of $0.1 \mathrm{eV}$. The FTIR spectra were recorded in the range $550-4000 \mathrm{~cm}^{-1}$ at room temperature using a Thermo Scientific Nicolet iS10. Attenuated total reflection (ATR) FTIR mode was used to obtain the reflectance infrared spectra of the polymer film. We signal-averaged 32 scans to form a single spectrum, which was then displayed in terms of transmittance. The baseline was corrected using OMNIC FTIR software. For the UV-vis measurements, the polymer film was electrochemically deposited onto an ITO-coated glass substrate, and the spectrum was acquired using a UV-vis spectrometer (OceanOptics USB 2000+) in $0.1 \mathrm{M} \mathrm{NaCl}$.

2.6. OECT Fabrication, Characterization, and Integration with SCs. The OECTs were fabricated on glass wafers (D $263 \mathrm{~T}$ eco, Schott) using photolithography based on a previous protocol. ${ }^{10}$ The device fabrication started with the patterning of $\mathrm{Au}$ contacts and interconnects. The photoresist S1813 was deposited on cleaned substrates followed by exposure to UV light using a contact aligner and development with an MF-319 developer. A $10 \mathrm{~nm}$ layer of $\mathrm{Cr}$ and a $100 \mathrm{~nm}$ layer of Au were then sputtered on the substrates and patterned the metal through a standard lift-off process. The second layer of photoresist AZ9260 was spin-coated on the substrate and developed using an AZ developer to define the channel areas. The channel patterns $($ width $=100 \mu \mathrm{m}$, length $=10 \mu \mathrm{m}$, thickness $=170$ 
$\mathrm{nm})$, and the contact pads were opened via reactive ion etching. PEDOT:PSS (PH 1000) was mixed with EG (5 vol \%), DBSA (0.002 vol \%), and GOPS (1 wt \%) and sonicated for $30 \mathrm{~min}$. The dispersion was then filtered through $0.45 \mu \mathrm{m}$ glass fiber filters and spin-coated on the channel at $3000 \mathrm{rpm}$ for $45 \mathrm{~s}$. After spin-coating, the sacrificial Parylene-C layer $(\sim 1.5 \mu \mathrm{m})$ was peeled off, and the films were annealed at $140{ }^{\circ} \mathrm{C}$ for $1 \mathrm{~h}$ in ambient conditions.

We connected an SC to a $\mathrm{Ag} \mid \mathrm{AgCl}$ pellet, which was used as the gate electrode of the OECT comprising PEDOT:PSS channels. The OECT gated with an aqueous salt solution $(100 \mu \mathrm{L}$ of $0.1 \mathrm{M} \mathrm{NaCl})$. We switched OFF/ON the OECT by inserting/taking out the gate electrode ( $\mathrm{Ag} \mid \mathrm{AgCl}$ pellet) into/from the electrolyte. Either a sourcemeasure unit (Keithley 2602A) or a second SC was used to supply voltage to the channel $\left(V_{\mathrm{D}}\right)$. The voltage provided by the $\mathrm{SC}$ was determined by measuring the open-circuit voltage (OCV) of the SC, which varied as a function of the SC charging rate. When SCs powered the OECT, an external load was integrated into the circuit to slow down the decay of the SCs' voltage output. For the $\mathrm{Na}^{+}$ionsensing experiment, the $\mathrm{Ag} \mid \mathrm{AgCl}$ pellet gate electrode was exchanged with a polyimide/ $\mathrm{Au}$-coated electrode functionalized with the sodium selective conducting polymer $\mathrm{p}(\mathrm{T} 15 \mathrm{c} 5$-ran-2EDOT $)$ reported in ref 54.

\section{RESULTS AND DISCUSSION}

We chose to electropolymerize EDOTOH on $\mathrm{Au}$ electrodes because electropolymerization is a robust and easy method to yield conducting polymer (CP) films on substrates of any given geometry. ${ }^{45}$ The fact that many properties of an electropolymerized polymer (nano- and microscale morphology, thickness, porosity, conductivity, and capacitance) can be controlled by varying the polymerization conditions and components enables the formation of a plethora of CPs. ${ }^{55,56}$ The monomer oxidation initiates the polymerization followed by a progressive color change of the electrode, indicating the accumulation of polymerized chains (see Scheme S1 for the polymerization route). The dioxy bridge in EDOTOH protects and stabilizes the aromatic thiophene ring from irreversible oxidation. We electropolymerized $\mathrm{p}(\mathrm{EDOTOH})$ on top of $\mathrm{Au}-$ coated flexible polyimide substrates in the presence of $\mathrm{ClO}_{4}{ }^{-}$ counterions in water at a constant potential of $1 \mathrm{~V}$ vs $\mathrm{Ag} \mid \mathrm{AgCl}$ (corresponding to a stabilized current of $0.43 \mathrm{~mA}$ ). A typical chronoamperometric curve recorded during the polymerization of $\mathrm{p}(\mathrm{EDOTOH}): \mathrm{ClO}_{4}{ }^{-}$is shown in Figure S1a. The chronoamperogram suggests that polymerization started very rapidly, characterized by a sharp increase in the current, followed by a progressive decline and a stabilization phase when the polymerization rate became equal to the monomers' arrival rate at the electrode through their migration or diffusion in the bulk. ${ }^{57}$ From these curves, we calculated the charge consumed in the polymerization process $\left(Q_{\mathrm{pol}}\right) . Q_{\mathrm{pol}}$ scales linearly $\left(R^{2}>0.99\right)$ with the mass of the film formed on the substrate $(W)$, corroborating the Faradaic process during the anodic polymerization (Figure 1a). From the slope of this linear curve, we extracted the current productivity ( $\left.i_{\text {productivity }}\right)$, expressed as milligrams of electrogenerated polymer per Coulomb of charge consumed during the electropolymerization. The $i_{\text {productivity }}$ under static conditions is equal to $0.75 \mathrm{mg}$ $\mathrm{C}^{-1}$, similar to the previously reported values for identical concentrations of EDOT used in the polymerization of PEDOT (i.e., $0.875 \mathrm{mg} \mathrm{C}^{-1}$ ), ${ }^{57}$ manifesting that the electrochemical reaction produces adequate linkages into electrogenerated polymeric chains, giving rise to a cross-linked network of $\mathrm{p}(\mathrm{EDOTOH})$ chains. $^{58}$ After a $5 \mathrm{~min}$ electrodeposition, hydrophilic and homogeneous films with a thickness of $\sim 500 \mathrm{~nm}$ were generated on top of flexible polyimide substrates (Figure $1 \mathrm{~b}-\mathrm{d}$ ). The films showed a globular surface morphology with nanometer-sized grains, leading to a root-mean-square (RMS) roughness of $\sim 28 \mathrm{~nm}$, as depicted from the SEM (Figure 1e) and AFM images (Figure $\mathrm{S} 1 \mathrm{~b})$. These clusters seem to arise from the aggregation of linear segments of polymer chains, increasing the roughness and porosity of the film surface, as observed in previous studies. ${ }^{59}$ Fourier transform IR (FTIR) spectroscopy was used to further validate the structure of the polymer film (Figure S1c). The bands in the spectrum of the EDOT monomer ascribed to the $\mathrm{C}-\mathrm{H}$ bending mode $\left(892 \mathrm{~cm}^{-1}\right)$ and the $\mathrm{C}-\mathrm{H}$ vibration (2879 and $2969 \mathrm{~cm}^{-1}$ ) disappear in the $\mathrm{p}$ (EDOTOH) spectrum, indicating the formation of PEDOT chains with $\alpha, \alpha^{\prime}$-coupling. ${ }^{60}$ In the polymer spectrum, the vibration bands appearing at 1508 and $1303 \mathrm{~cm}^{-1}$ are ascribed to the stretching mode of $\mathrm{C}=\mathrm{C}$ and inter-ring stretching mode of $\mathrm{C}-\mathrm{C}$ in the thiophene chain, respectively. The characteristic band of the stretching vibration of the $\mathrm{C}-\mathrm{S}-\mathrm{C}$ bond in the thiophene chain is also observed at 696,823 , and $991 \mathrm{~cm}^{-1}$, confirming the successful formation of the PEDOT derivative upon the electropolymerization reaction. ${ }^{61,62}$ Additionally, the UV-vis absorption spectra (Figure S1d) of the electropolymerized polymer on top of an ITO substrate reveal a broad absorption band starting from $700 \mathrm{~nm}$ extending to the NIR region. The broad peak at 400 to $800 \mathrm{~nm}$ is attributed to the $\pi-\pi^{*}$ transition of thiophene chains, while the band at $\sim 1060 \mathrm{~nm}$ is credited to polaron and/or bipolaron bands, characteristic of oxidized PEDOT. ${ }^{63,64}$

Figure 1f shows a typical CV curve of the $\mathrm{p}(\mathrm{EDOTOH})$ film. The potential window applied to the film $(-0.5$ to $0.7 \mathrm{~V})$ was chosen to avoid the interference from the oxygen evolution reaction during the anodic scan and hydrogen evolution on the cathodic scan. The non-perfect rectangular shape (i.e., fill factor $(\mathrm{FF})=77 \%$ ) stems from the non-vertical shift in current density upon altering the electrode polarity near -0.5 and $0.7 \mathrm{~V}$, indicating polarization resistance. ${ }^{65}$ The CV curve shape did not change upon 1000 cycles, with a small reduction in the current magnitude (FF drops from 77 to $73 \%)$. Moreover, we observed that, with an increase in the scan rate, the film produces larger current densities (Figure S2a). Plotting the current density at a non-Faradaic region against the scan rate (Figure S2b), we found the double-layer/areal capacitance of the $\mathrm{p}(\mathrm{EDOTOH})$ electrode to be $9.15 \mathrm{mF} \mathrm{cm}$ (eq 1), up to par with other reported freestanding PEDOTonly films. ${ }^{42,66}$ Analogous results were found in galvanostatic charge-discharge (GCD) cycling conditions, which continuously oxidize and reduce the film at a constant current (Figure $1 \mathrm{~g}$ ). At a normalized current density of $1.3 \mathrm{~A} \mathrm{~g}^{-1}$, we obtained typical triangular charge-discharge curves, leading to a specific capacitance of $75 \mathrm{~F} \mathrm{~g}^{-1}$. As we cycle the film, anions are transferred to the film during oxidation and released back into the solution while the counterions (i.e., cations) penetrate the film during reduction. Hence, the charge-discharge process in the CP film takes place throughout the bulk volume of the film and not just on the surface. ${ }^{67}$ Such volumetric ion penetration can be detrimental to the film stability. We therefore implemented charge-discharge cyclic conditions for 1000 cycles to investigate the stability of the $\mathrm{p}(\mathrm{EDOTOH})$ film against volumetric ion penetration. The capacitance retention after 1000 cycles was $\sim 82 \%$ (Figure $1 \mathrm{~g}$ ). The Nyquist plots of the film before and after cycling (Figure S2c,d) suggest that the loss of capacitance upon cycling is due to the reduced doublelayer capacitance and bulk capacitance of the film (Table S1). 
(a)
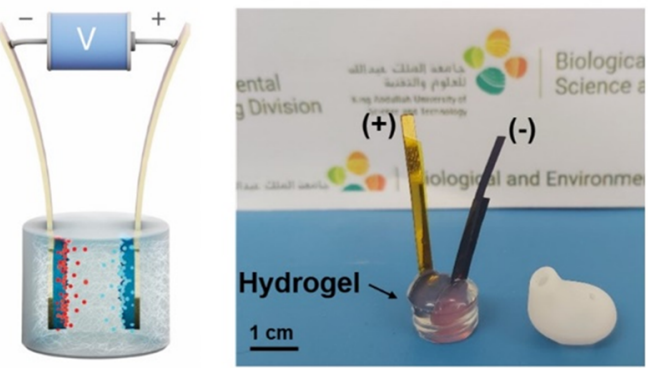

(c)

(e)
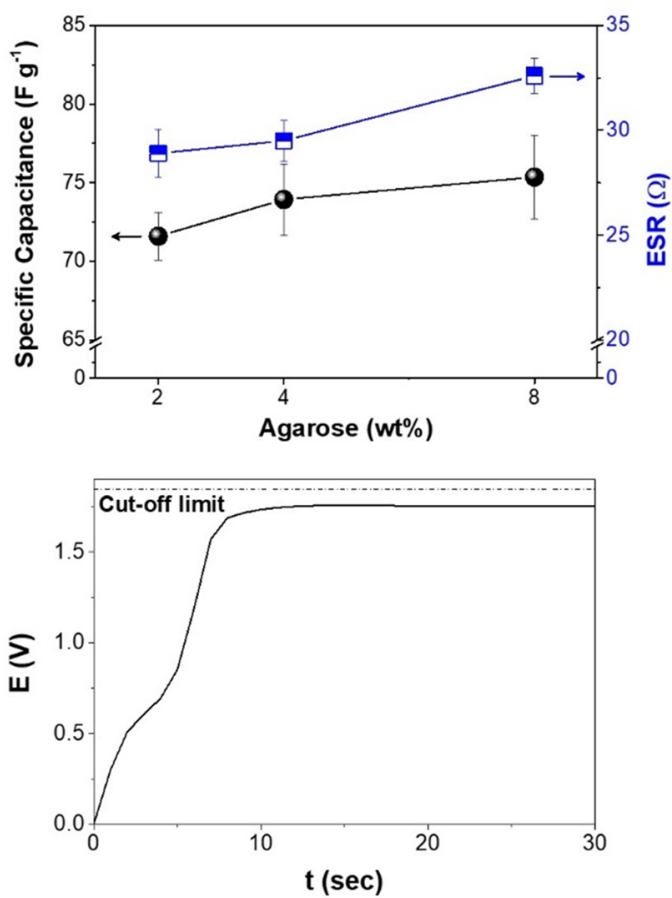

(b)

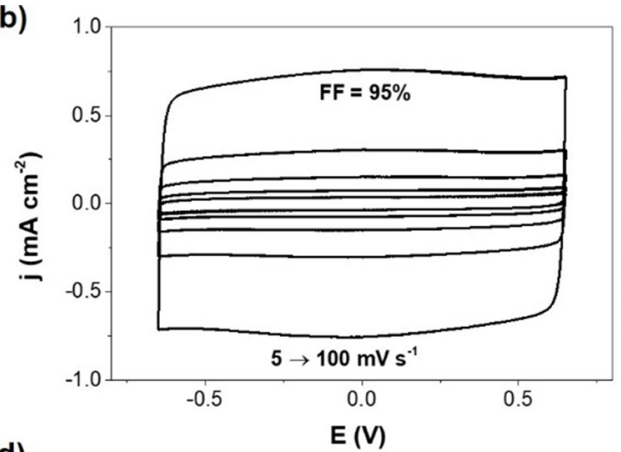

(d)

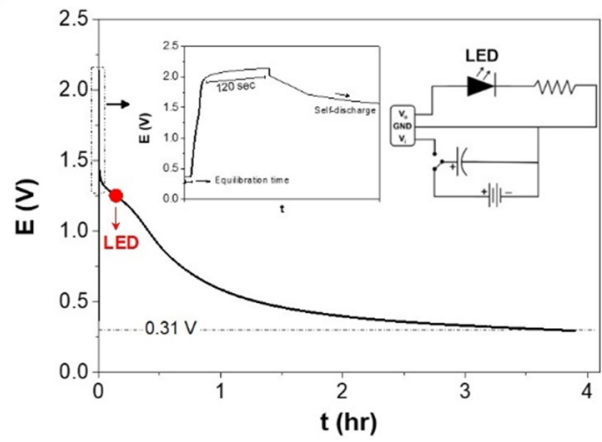

(f)

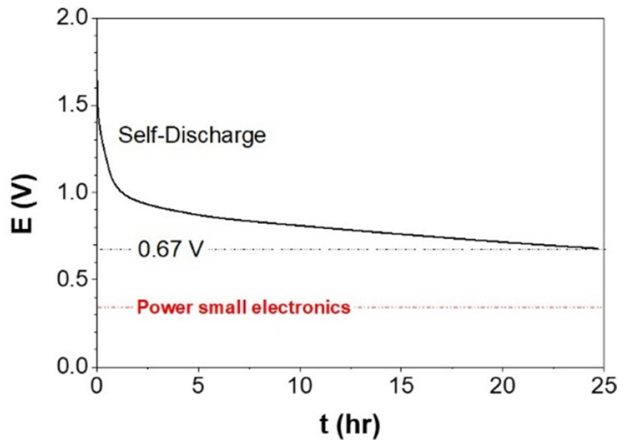

Figure 2. All-polymer self-standing hydrogel SC. (a) Schematic of the SC along with an optical picture of the SC next to an earplug. (b) CV curves of the SC acquired at different scan rates in $0.1 \mathrm{M} \mathrm{NaCl}$ in ambient conditions; FF: fill factor. (c) Specific capacitance and equivalent series resistance (ESR) of the SC as a function of agarose weight percent that it comprises. The current density was $8.47 \mathrm{~A} \mathrm{~g}^{-1}$. The error bars are calculated from three separate SC devices. (d) Discharge voltage profile over time. The current density was $3.44 \mathrm{~A} \mathrm{~g}^{-1}$. LED denotes the time when the SC was connected to the LED. Inset shows the charging/discharging profile of the device at $t<5$ min (left), and the electronic circuit comprising the SC powering the LED (right). (e) Short-term potentiometry of the SC at a current density of $6.89 \mathrm{~A} \mathrm{~g}^{-1}$ (i.e., $0.5 \mathrm{~mA}$ ). (f) Selfdischarge curve of the SC under an open-circuit condition for $24 \mathrm{~h}$ after being charged at $1.75 \mathrm{~V}$ in $(\mathrm{e})$. The device was wetted once every $4 \mathrm{~h}$, with $0.2 \mathrm{~mL}$ of $0.1 \mathrm{M} \mathrm{NaCl}$.

In addition, the pronounced semicircle response (inset of Figure S2c) indicates a higher interfacial charge transfer resistance after cycling. ${ }^{68}$

The second component of our SC is a hydrogel whose properties are critical to the device performance. Hydrogels are $3 \mathrm{D}$, cross-linked polymeric networks able to absorb and retain aqueous solutions many times over their weight without losing structural integrity and getting dissolved. ${ }^{69}$ Agarose is a naturally occurring polysaccharide composed of linear chains of agarobiose, which consists of D-galactose and 3,6-anhydro-Lgalactopyranose. ${ }^{70}$ The gelation of agarose involves the crosslinking and self-assembly of molecules via hydrogen bonding. ${ }^{71}$ The resulting hydrogel is known for its thermoreversible gelling property, porosity, hydrophilicity, low cost, and high ionic conductivity. ${ }^{70,72}$ To find out the optimal agarose hydrogel properties (e.g., powder content, thickness of the gel) and evaluate the performance of the electropolymerized $\mathrm{p}(\mathrm{EDOTOH})$ films in a two-electrode system, we first built an
$\mathrm{H}$-cell where the hydrogel is used as the separation membrane (Figure S3a).

This H-cell could accommodate a range of current densities as low as $0.84 \mathrm{~A} \mathrm{~g}^{-1}$ to as high as $17 \mathrm{~A} \mathrm{~g}^{-1}$ (Figure S3b). We found that a hydrogel thickness of $2.26 \mathrm{~mm}$ at an agarose weight percentage of $8 \%$ yielded the best-performing capacitance retention, greater than $95 \%$ for 4000 galvanostatic charge-discharge cycles at $3.4 \mathrm{~A} \mathrm{~g}^{-1}$ (Figure S3c). On the other hand, a 10-fold increase in the $\mathrm{NaCl}$ concentration of the operating medium did not cause a significant change in the cell's performance, suggesting that the porous network retains enough ions for conduction at $0.1 \mathrm{M} \mathrm{NaCl}$ (Figure S3d). At the same time, inferior specific capacities were recorded in the presence of more conventional but less hydrophilic membranes such as Nafion and cellulose (Figure S3d). However, the agarose $\mathrm{H}$-cell revealed high equivalent series resistance (ESR), as gleaned from the charge-discharge curves (Figure S3e). ESR describes the overall resistance of the cell and is a crucial 
factor determining the power density and energy of the SC. ${ }^{36}$ High ESR primarily stems from polymer degradation and mechanical stress on the membrane. ${ }^{73}$ Since the electrochemical window chosen here does not allow for parasitic reactions, care was taken for minimal mechanical stress, the temperature was set in ambient conditions, and the electrolyte concentration was adequately high, we attribute the high ESR values to the distance between the anode and cathode electrodes in this particular device geometry (i.e., $2 \mathrm{~cm}$, Figure S3a). Note also that the ESR is independent of agarose thickness, which was varied between 0.6 and $4.3 \mathrm{~mm}$ (Table S2). These results advocate for a different architecture that ensures a more compact configuration for a high-performance SC.

Our results with the $\mathrm{H}$-cell suggest that instead of using the gel as a membrane, the SC electrodes should be assembled all together within the hydrogel, as depicted in Figure 2a. A compact, self-standing SC was generated in situ while the agarose solution solidified around the electrodes (see Scheme 1 for the assembly procedure). The process of forming the hydrogel is simple (i.e., no functionalization is required), reproducible, and fast (i.e., the hydrogel can be prepared in 20 min under laboratory conditions). Figure $2 \mathrm{a}$ shows a photograph of the self-standing hydrogel SC. The distance between the anode and the cathode in this configuration markedly decreased to $1.5 \mathrm{~mm}$, required for low ESR. The scan rate-dependent CV curves of the hydrogel SC (8 wt \% agarose) reveal a rectangular pattern (Figure $2 \mathrm{~b}$ ), typical of an electrical double-layer capacitor. The similar shape of this $\mathrm{CV}$ curve to that of the electrodes measured in the electrolyte (Figure 1f) highlights that the hydrogel pore diameter is large enough (i.e., $150 \mathrm{~nm}$, see Figure S4) to allow for the transport of ions therein. Moreover, the device retained its rectangular shape even at high scan rates $\left(100 \mathrm{mV} \mathrm{s}^{-1}\right)$ as well as elevated temperatures (i.e., mimicking the physiological temperature of $37{ }^{\circ} \mathrm{C}$, Figure S5), signifying a fast charge storage mechanism independent of speed of voltage applied or temperature. The $\mathrm{CV}$ curves reveal an FF of $\sim 95 \%$, calculated at $100 \mathrm{mV} \mathrm{s}^{-1}$, demonstrating a highly symmetrical charge/discharge behavior of the SC. These results have been anticipated as agarose gels were reported to operate with charged species larger than hydrated $\mathrm{Na}^{+}$or $\mathrm{Cl}^{-}$, for example, with DNA for electrophoresis applications. ${ }^{73}$ We next estimated the specific capacitance of the all-polymer self-standing hydrogel SC (8 wt \% agarose) to be $75 \mathrm{~F} \mathrm{~g}^{-1}$ at a current density of $8.47 \mathrm{~A} \mathrm{~g}^{-1}$ and a double-layer capacitance of $\sim 3.7 \mathrm{mF} \mathrm{cm}^{-2}$. The specific capacitance value was only marginally affected by agarose weight percentage, as depicted in Figure 2c. Most importantly, the SC had a 10 -fold decrease of the ESR (from 340 to $32 \Omega$ ) compared to the $\mathrm{H}$-cell configuration (Figure S3a). We attribute the low ESR value to the compactness of the new device architecture, which results in facile ionic transport (i.e., efficient pathways to the $\mathrm{p}(\mathrm{EDOTOH})$ sites due to improved interfacial contact) with excellent isolation of electronic flow. In addition, the SC operated similarly when the gel was fed with aqueous solutions other than PBS, such as $\mathrm{KCl}$ and $\mathrm{NaNO}_{3}$ (Figure S6a,b, respectively), evidencing its versatility toward the use of other aqueous salts.

We next evaluated the performance of the SC as a circuit element. The SC charged with $3.44 \mathrm{~A} \mathrm{~g}^{-1}$ for $30 \mathrm{~s}$ readily activated a red LED upon self-discharge (Figure $2 \mathrm{~d}$ ). At the same time, the open-circuit potential stabilized after $4 \mathrm{~h}$ at $\sim 0.31 \mathrm{~V}$, a voltage output large enough to power various bioelectronic devices (Figure $2 \mathrm{~d}$ ). The slow discharge time between 2 and $4 \mathrm{~h}$ renders the device suitable as a continuous power supply. In addition, in the absence of the LED and by loading with higher charging currents (i.e., for $30 \mathrm{~s}$ at $6.89 \mathrm{~A}$ $\mathrm{g}^{-1}$, see charging profile in Figure 2e), the self-discharge lasted for over 1 day, provided that the device was wetted once every $4 \mathrm{~h}$ (Figure 2f). The open-circuit potential of this device reached $0.67 \mathrm{~V}$ after $25 \mathrm{~h}$, with a $36 \mathrm{mV}$ decrease per hour (measured $4 \mathrm{~h}$ after the charging). The main contributors of the potential drop under self-discharge are (i) Faradaic reactions with either activation-controlled or diffusioncontrolled mechanism, (ii) ohmic leakage, and (iii) charge redistribution. ${ }^{74-76}$ Leakage current can occur due to redox reactions, ionic charge diffusion, and/or partial electronic discharge through the separator. The corresponding diagnostic test (Figure S7a) revealed a linear dependency of the voltage drop on the logarithm of time, signifying that the $60 \%$ decline within $25 \mathrm{~h}$ stems from a Faradaic self-discharge initiated by overcharge. ${ }^{77}$ The leakage current extracted from Figure $2 \mathrm{f}$ was of the order of $1.65 \times 10^{-5} \mathrm{~mA}$ (Figure S7b). These experiments suggest that the device can supply enough voltage for extended periods to low-power bioelectronics as long as it is in a wet environment, which makes it particularly suitable for use in an in vivo setting.

Next, we sought to evaluate the stability of the device upon continuous cycling. Figure 3 a displays the variation in specific capacitance and Coulombic efficiency of the SC as a function of cycle number for two different current densities, 3.44 and $6.89 \mathrm{~A} \mathrm{~g}^{-1}$. Capacitance retentions of 75 and $71 \%$ of the devices' initial capacitance were recorded after 12,000 cycles, indicating good rate capability, associated with the efficient ion transport from the electrolyte to the electrode. Meanwhile, the Coulombic efficiency remained at $100 \%$, further manifesting the robust performance of the device. Nevertheless, we explored further the source of the capacitance loss upon cycling as such analysis could inform which film properties should be improved in the long run and which operation parameters should be avoided. Electrochemical impedance spectroscopy measurements performed before and after cycling revealed sluggish mass transport and a decrease of the capacitance for the cycled SCs.

Figure S8 denotes the variation of capacitance with frequency, indicative of the electrolyte ion penetration inside the $\mathrm{p}(\mathrm{EDOTOH})$ film at each frequency. The value of the real part of the capacitance at low frequencies reflects the bulk capacitance of the SC. Here, we measure a $20 \%$ decrease after the film was cycled for 12,000 times, possibly due to an electrochemical degradation mechanism. ${ }^{78}$ Furthermore, we observed a reduction in the dielectric relaxation time constant (i.e., $\tau_{\mathrm{o}}=3.03 \mathrm{~s}$ vs $\tau_{\mathrm{o}}=1.04 \mathrm{~s}$, see Figure S5b) as the films were cycled, suggesting hindered mass transport. The post-mortem analyses of the SC components showed signs of deterioration with cycling, accounting for this loss in capacitance. A roughened morphology after cycling $(12,000$ cycles) is evident from the SEM images, which shows the formation of micrometer-size clusters (between 1 and $5 \mu \mathrm{m}$ ) on the surface of the films (Figure S9a,b). We recorded the high-resolution carbon (C 1s) XPS spectra of the pristine $\mathrm{p}(\mathrm{EDOTOH})$ electrode as well as those after cycling. Figure S9c shows the typical XPS spectrum of the pristine sample, deconvoluted into three peaks, including the $\mathrm{C}-\mathrm{C} / \mathrm{C}=\mathrm{C}$ bonds at $284.5 \mathrm{eV}, \mathrm{C}-$ $\mathrm{O} / \mathrm{C}-\mathrm{S}$ bonds at $286 \mathrm{eV}$, and $\mathrm{C}=\mathrm{S}$ bond at $288 \mathrm{eV}$. ${ }^{79} \mathrm{We}$ found, however, an additional peak in the spectrum of the 
(a)

$3.44 \mathrm{~A} \mathrm{~g}^{-1}$

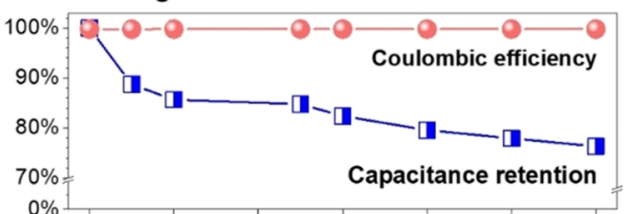

$6.89 \mathrm{Ag}^{-1}$

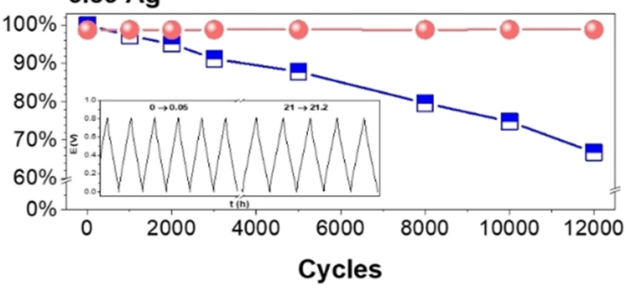

(b)

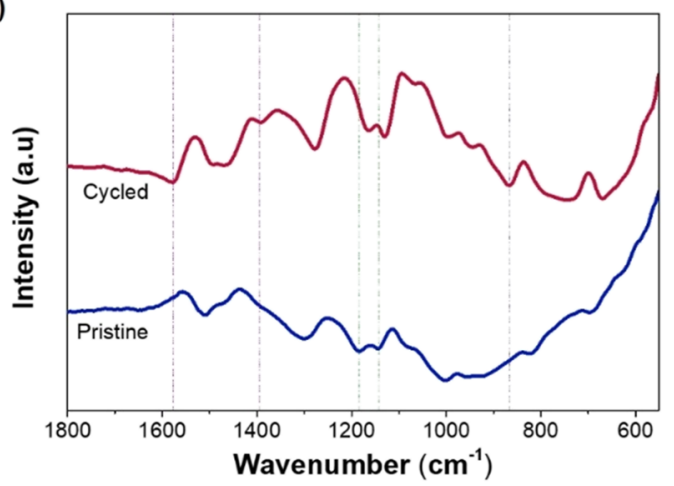

(c)

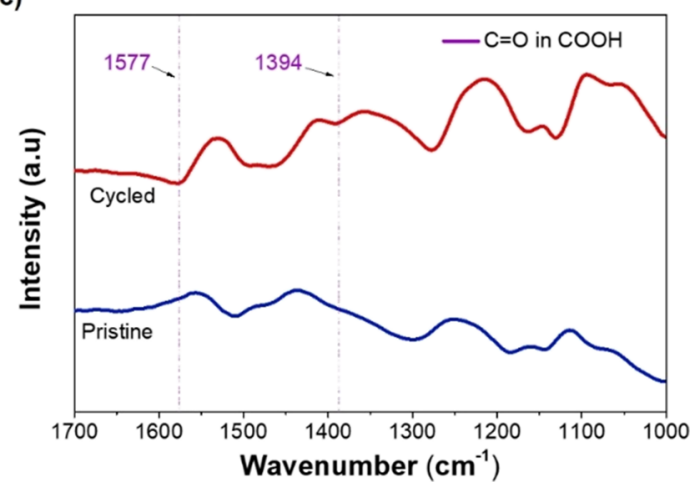

Figure 3. (a) Specific capacitance retention and Coulombic efficiency of the self-standing hydrogel SC upon 12,000 cycles for two different current densities. Inset shows the GCD response for six consecutive cycles of the device during various stages of the cycling test. (b, c) ATR-FTIR spectrum of the pristine and cycled (12,000 times, at a current density of $\left.6.89 \mathrm{~A} \mathrm{~g}^{-1}\right) \mathrm{p}($ EDOTOH) film. Full spectrum in the range of $550-1800 \mathrm{~cm}^{-1}$ (top) along with the magnified spectrum to show the characteristic peaks of $\mathrm{C}=\mathrm{O}$ stretching vibrations (bottom).

cycled cathode $(5.89 \%)$ and anode $(6.11 \%)$, originating from carboxylate groups (Figure S9d,e). This peak suggests a partial overoxidation of the $\mathrm{p}(\mathrm{EDOTOH})$ electrode upon cycling, possibly due to the cleavage of the $\mathrm{C}-\mathrm{C}$ bond, which is converted into a carboxylic group. ${ }^{80}$ The decrease of the C$\mathrm{C} / \mathrm{C}=\mathrm{C}$ bond contribution (from 56.2 to 48.5 and $51.5 \%$ for the cathode and anode, respectively) further corroborates the electrochemical degradation of the CP electrodes (see Table S3 for a summary of XPS signals).

Lastly, these changes are not only confined on the surface of the film, but they are also seen in the bulk of the cycled films.
ATR-FTIR spectra in Figure 3b reveal two new bands at 1394 and $1577 \mathrm{~cm}^{-1}$ for the cycled films, stemming from the stretching of the carboxyl group, ${ }^{81}$ attributed to the overoxidation of the PEDOT. Electrochemical degradation can lead to chemical bonds in CPs to be broken, leading to the polymer chains being irreversibly overoxidized into fragments. ${ }^{78} \mathrm{~A}$ band at $866 \mathrm{~cm}^{-1}$ appeared with long-term cycling, attributed to the vibrations of the sulfone group after a part of the thiophene units becomes overoxidized (Figure S10a). Moreover, the shift of the $\mathrm{C}-\mathrm{O}-\mathrm{C}$ bands at 1142 and 1183 $\mathrm{cm}^{-1}$ (Figure $\mathrm{S} 10 \mathrm{~b}$ ) advocates a change in the electrode's molecular vibrations as the bonds become less stiff and require lower energy. The optimization of operating conditions such as choosing a narrower range for the applied potential and/or current and slower scan rate could avoid the overoxidation of PEDOT electrodes.

An important trait of our capacitor is its flexible form factor thanks to the easy assembly of its components. In the experiments above, the SC had a weight of $1.95 \mathrm{~g}( \pm 0.12 \mathrm{~g})$, and the gel had a volume of $0.5 \mathrm{~mL}$. These dimensions can further go down as we built a miniaturized version of the device with the active area of the anode and cathode being equal to $0.053 \mathrm{~cm}^{2}$, leading to a lightweight, spherical SC $\left(W_{\text {total }}=0.7 \mathrm{~g}, \pm 0.04 \mathrm{~g}\right)$ with a volume of $0.065 \mathrm{~cm}^{3}\left(V_{\text {hydrogel }}=\right.$ $0.2 \mathrm{~mL}$ ), as illustrated in Figure $4 \mathrm{a}$ and further in Figure S11. The specific capacitance of this "micro-SC" was indistinguishable to the larger SC (shown in Figure 2c) and decreased with increasing current density (Figure $4 \mathrm{~b}$ ) owing to the increase of charge transfer resistance of $\mathrm{p}(\mathrm{EDOTOH}) .{ }^{82}$ The energy density that the micro-SC provides is between 15 and $20 \mathrm{~W}$ $\mathrm{kg}^{-1}$, while the power density reaches a maximum of $70 \mathrm{kWh}$ $\mathrm{kg}^{-1}$ for a discharge time of $0.75 \mathrm{~s}$. The high values of the power density (i.e., how fast the ions can move) are well aligned with surge-power delivery applications and, in turn, small (bio)electronics. The power characteristics of the miniaturized device are illustrated in the Ragone plot in Figure 4c. The performance of the miniaturized all-polymer SC is up to par with other reported systems based on PEDOT electrodes. The notable stability of the SC combined with its high power density and flexible geometry renders this system a promising candidate for both in vivo and in vitro energy storage and delivery applications. As a final point, the microSC can deliver a volumetric capacitance of $240 \mathrm{~F} \mathrm{~cm}^{-3}$ at a current density of $1.07 \mathrm{~A} \mathrm{~g}^{-1}$ in line with other PEDOT-based supercapacitor devices (Table S4) and can afford a capacitance of $200 \mathrm{~F} \mathrm{~cm}^{-3}$ at $28.56 \mathrm{~A} \mathrm{~g}^{-1}$.

As we verified the performance and stability of the selfstanding SC and used it to power an LED, we next integrated it with a microscale OECT. We connected the SC to a $\mathrm{Ag} \mid \mathrm{AgCl}$ pellet, which was used as the gate electrode of the OECT comprising PEDOT:PSS-coated channels (width $=100 \mu \mathrm{m}$, length $=10 \mu \mathrm{m}$, thickness $=170 \mathrm{~nm})$. Our OECT is electrolyte-gated, meaning that it operates in an aqueous salt $(100 \mu \mathrm{L}$ of $0.1 \mathrm{M} \mathrm{NaCl})$. Figure 5a shows a cross-sectional schematic of an OECT, and a photograph of a chip comprising six identical channels is given in Figure S12. In the absence of a gate voltage $\left(V_{\mathrm{G}}\right)$, the PEDOT:PSS channel is in the ON state since PEDOT:PSS is an inherently oxidized polymer. As a positive $V_{\mathrm{G}}$ is applied throughout the aqueous electrolyte, the channel is depleted by cations penetrating the film. At the same time, holes are extracted at the drain contact to maintain charge balance. The net result of gating in this "depletion mode" OECT is a decrease in the channel current. Figure $5 \mathrm{~b}$ 
(a)

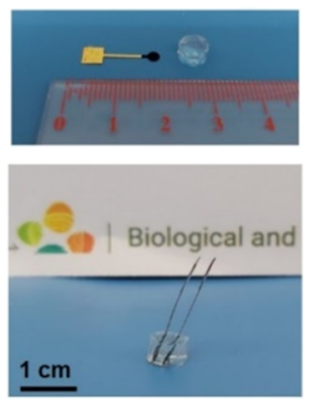

(b)

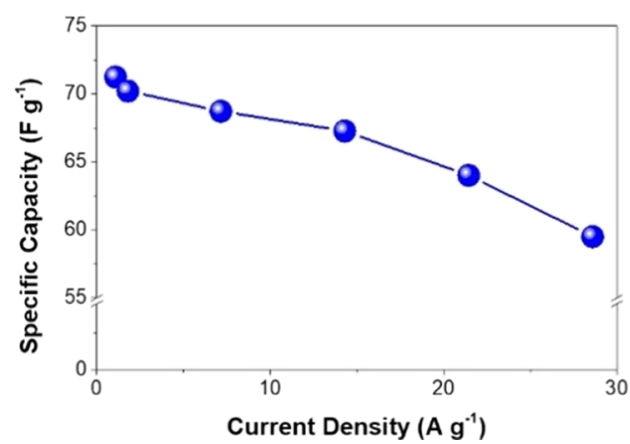

(c)

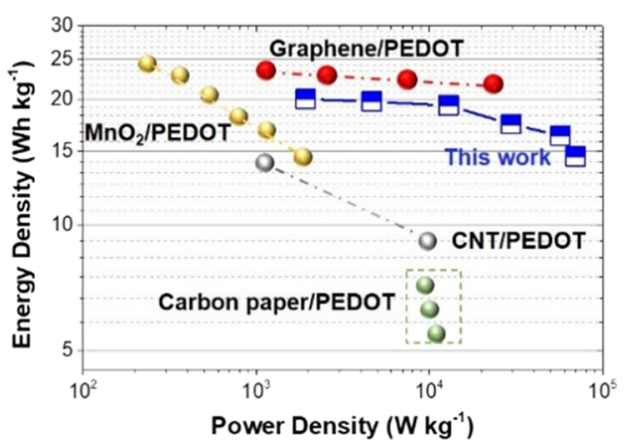

Figure 4. Miniaturized self-standing hydrogel SC (micro-SC). (a) Photograph depicting the dimension and shape of the components and the assembled micro-SC. The circular active area of the anode and cathode is $\sim 0.053 \mathrm{~cm}^{2}$. (b) Specific capacitance vs current density of the micro-SC. (c) Comparative Ragone plots of the self-standing hydrogel SC with other PEDOT-based SCs, including $\mathrm{MnO}_{2} / \mathrm{PEDOT}^{83}$ carbon paper/ PEDOT $^{29} \mathrm{CNT}^{\mathrm{P}} \mathrm{PEDOT}^{84}$ and graphene/PEDOT. 85

(a)

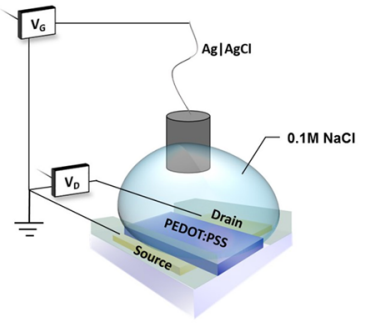

(c)

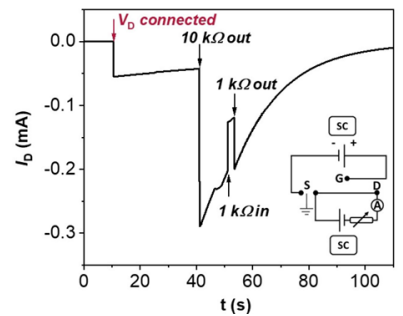

(b)

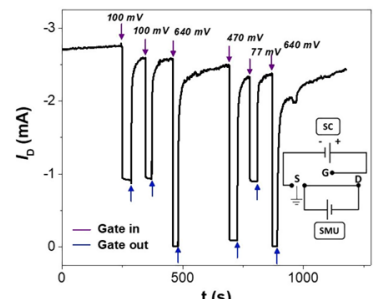

(d)

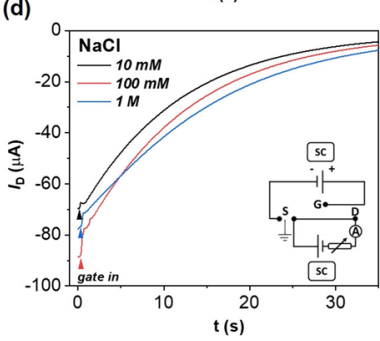

Figure 5. (a) Schematic of a PEDOT:PSS OECT gated with a Agl $\mathrm{AgCl}$ electrode in $0.1 \mathrm{M} \mathrm{NaCl}$. The channel has a width of $100 \mu \mathrm{m}$, length of $10 \mu \mathrm{m}$, and thickness of $170 \mathrm{~nm}$. (b) Real-time response of the OECT when the gate was connected to a self-standing SCs. Drain voltage $\left(V_{\mathrm{D}}\right)$ was $-0.6 \mathrm{~V}$. The voltage applied by the gate electrode was modulated by the open-circuit voltage of the SC connected to the gate. (c) Two SCs were used to supply the drain and the gate voltages. The circuit contained an external load (with varied resistances) to slow down the discharging rate. (d) Current response of a $\mathrm{Na}^{+}$-selective OECT channel to a change in $\mathrm{NaCl}$ concentration from $10 \mathrm{mM}$ to $1 \mathrm{M}$. The device was powered by two self-standing SCs. Insets show the equivalent electronic circuit connection of the polarized capacitor with the OECT. S, source; D, drain; G, ground; SMU, source-measure unit.

displays the current profile of a PEDOT:PSS channel where the gate is connected to the charged, all-polymer SC, monitored for $20 \mathrm{~min}$. We switch OFF/ON the OECT by inserting/taking out the gate electrode ( $\mathrm{Ag} / \mathrm{AgCl}$ pellet) into/ from the electrolyte. The gate potential acting on the semiconductor channel is proportional to the open-circuit potential of the discharging SC connected to the transistor gate. As the positive gate voltage supplied by the SC increased, the source-drain current $\left(I_{\mathrm{D}}\right)$ decreased, consistent with the depletion mode OECT operation. We used multiple SCs that were charged at different rates to simulate different gating potentials. Here, a source-measure unit was used to supply

voltage to the channel $\left(V_{\mathrm{D}}\right)$. As for the gate source, an SC can also supply the voltage for the source-drain. Figure $5 \mathrm{c}$ shows the channel readout as the SC was connected to the source and drain contact with an output voltage of $-0.10 \mathrm{~V}$. The circuit contained an external load to slow down the decay of the voltage output of the SC to have stable drain current outputs over time. Once this load was disconnected, the current first increased and then decayed to lower values as the SC discharged. The discharge rate of the SC and the voltage it supplies to a circuit can be easily manipulated by the integration of such circuit elements.

To further demonstrate the use of the SC in an actual biosensor circuit, we built a $\mathrm{Na}^{+}$sensor. For these experiments, we polymerized ion-selective crown-ether-functionalized EDOT-based films as has recently been reported in our group. ${ }^{54}$ The OECTs comprising the $\mathrm{Na}^{+}$-selective copolymer at the gate electrode was shown to have channel current scaling with $\mathrm{Na}^{+}$content of the electrolyte. ${ }^{54}$ We deposited the $\mathrm{Na}^{+}-$ selective copolymer on the laterally patterned gate electrode of the OECT (Figure S12). The channel was based on PEDOT:PSS and had a geometry identical to the one used in the experiments above. We monitored the response of this OECT in aqueous solutions containing different $\mathrm{NaCl}$ concentrations while both the drain and gate contacts were connected to two discharging SCs. The supercapacitor upon discharge will release its energy as electric current into the OECT. The $V_{\mathrm{G}}$ and $V_{\mathrm{D}}$ are supplied by two separate SCs with an output voltage of -0.2 and $-0.3 \mathrm{~V}$, respectively, after charging them for $1 \mathrm{~min}$ at $3.44 \mathrm{~A} \mathrm{~g} \mathrm{~g}^{-1}$. Figure $5 \mathrm{~d}$ shows the current profiles of this SC-powered ion sensor in different electrolytes. Note that as the discharging kinetics is preset for the SCs, the voltage that they supply to the OECT gate and channel remains the same for the measurements in three electrolytes. The drain current monitored for $30 \mathrm{~s}$ is higher in the concentrated electrolytes compared to the dilute one $\left(I_{\mathrm{D} \_1 \mathrm{M}}>I_{\mathrm{D} \_100 \mathrm{mM}}>I_{\mathrm{D} \_10 \mathrm{mM}}\right)$, in agreement with the operation of this type of ion sensor.

\section{CONCLUSIONS}

The growing interest in bioelectronics has to be capitalized with compliant powering devices. Powering a biosensor/ actuator is of paramount importance for the viability of such devices in everyday life applications. To this end, we presented a simple and straightforward strategy to devise a lightweight, 
biocompatible, and cost-effective supercapacitor made from electropolymerized $\mathrm{p}(\mathrm{EDOT}-\mathrm{OH})$ films embedded in a selfstanding agarose hydrogel. The device has the advantage of continually utilizing an external source of biocompatible liquid for energy storage. The hydrogel acted as a mechanical support as well as a source of ions. This conducting polymer-based selfstanding hydrogel SC was robust (capacitance retention of $75 \%$ after 12,000 cycles) and exhibited high power density $\left(10^{5} \mathrm{~W} \mathrm{~kg}^{-1}\right)$, long self-discharge $(>2 \mathrm{~h})$, and fast response time (0.1-30 seconds) while it can afford a specific capacitance of $71 \mathrm{~F} \mathrm{~g}^{-1}$ and volumetric capacitance of $240 \mathrm{~F}$ $\mathrm{cm}^{-3}$. The device also possessed operational flexibility (can accommodate various types of aqueous electrolytes), and its geometry was easy to change. When miniaturized, the SC yielded similar power characteristics to larger prototypes. By further optimizing the electrode material, higher performances in terms of specific capacitance and power density can be realized for SCs that are easy to assemble in various forms and shapes compatible with biological media. Stemming from the growing interest in the design and engineering of implantable operational energy generating devices, this research contributes to the technological advancement of cost-effective energy storage devices that can power micron-scale biosensors/ actuators.

\section{ASSOCIATED CONTENT}

\section{SI Supporting Information}

The Supporting Information is available free of charge at https://pubs.acs.org/doi/10.1021/acsaem.0c01299.

Literature review, electropolymerization route, optical pictures and additional experimental results (AFM, CV, EIS) (PDF)

\section{AUTHOR INFORMATION}

\section{Corresponding Author}

Sahika Inal - Organic Bioelectronics Laboratory, Biological and Environmental Science and Engineering (BESE), King Abdullah University of Science and Technology (KAUST), Thuwal 23955-6900, Saudi Arabia; ○ orcid.org/0000-0002-11661512; Email: sahika.inal@kaust.edu.sa

\section{Authors}

Georgios Nikiforidis - Organic Bioelectronics Laboratory, Biological and Environmental Science and Engineering (BESE), King Abdullah University of Science and Technology (KAUST), Thuwal 23955-6900, Saudi Arabia

Shofarul Wustoni - Organic Bioelectronics Laboratory, Biological and Environmental Science and Engineering (BESE), King Abdullah University of Science and Technology (KAUST), Thuwal 23955-6900, Saudi Arabia

David Ohayon - Organic Bioelectronics Laboratory, Biological and Environmental Science and Engineering (BESE), King Abdullah University of Science and Technology (KAUST), Thuwal 23955-6900, Saudi Arabia

Victor Druet - Organic Bioelectronics Laboratory, Biological and Environmental Science and Engineering (BESE), King Abdullah University of Science and Technology (KAUST), Thuwal 23955-6900, Saudi Arabia

Complete contact information is available at: https://pubs.acs.org/10.1021/acsaem.0c01299

\section{Notes}

The authors declare no competing financial interest.

\section{ACKNOWLEDGMENTS}

G.N. and S.I. thank the support by the King Abdullah University of Science and Technology (KAUST) Office of Sponsored Research (OSR) under Award No. OSR-2015Sensors-2719. Scheme ${ }^{1}$, Figure ${ }^{2}$ a, and the TOC image were created by Heno Hwang, scientific illustrator at KAUST. The authors thank Dr. Craig Combe and Prof. Iain McCulloch (KAUST Solar Center) for the synthesis of the ion-selective EDOT monomer.

\section{REFERENCES}

(1) Berggren, M.; Richter-Dahlfors, A. Organic Bioelectronics. Adv. Mater. 2007, 19, 3201-3213.

(2) Song, Y.; Min, J.; Gao, W. Wearable and Implantable Electronics: Moving toward Precision Therapy. ACS Nano 2019, $13,12280-12286$.

(3) Someya, T.; Bao, Z.; Malliaras, G. G. The Rise of Plastic Bioelectronics. Nature 2016, 540, 379-385.

(4) Luo, S.-C. Conducting Polymers as Biointerfaces and Biomaterials: A Perspective for a Special Issue of Polymer Reviews. Polym. Rev. 2013, 53, 303-310.

(5) Inal, S.; Malliaras, G. G.; Rivnay, J. Benchmarking Organic Mixed Conductors for Transistors. Nat. Commun. 2017, 8, 1767.

(6) Rivnay, J.; Inal, S.; Salleo, A.; Owens, R. M.; Berggren, M.; Malliaras, G. G. Organic Electrochemical Transistors. Nat. Rev. Mater. 2018, 3, 17086.

(7) Inal, S.; Rivnay, J.; Suiu, A.-O.; Malliaras, G. G.; McCulloch, I. Conjugated Polymers in Bioelectronics. Acc. Chem. Res. 2018, 51, $1368-1376$

(8) Khodagholy, D.; Doublet, T.; Quilichini, P.; Gurfinkel, M.; Leleux, P.; Ghestem, A.; Ismailova, E.; Hervé, T.; Sanaur, S.; Bernard, C.; Malliaras, G. G. In Vivo Recordings of Brain Activity Using Organic Transistors. Nat. Commun. 2013, 4, 1575.

(9) Parlak, O.; Keene, S. T.; Marais, A.; Curto, V. F.; Salleo, A. Molecularly Selective Nanoporous Membrane-Based Wearable Organic Electrochemical Device for Noninvasive Cortisol Sensing. Sci. Adv. 2018, 4, No. eaar2904.

(10) Wustoni, S.; Hidalgo, T. C.; Hama, A.; Ohayon, D.; Savva, A.; Wei, N.; Wehbe, N.; Inal, S. In Situ Electrochemical Synthesis of a Conducting Polymer Composite for Multimetabolite Sensing. Adv. Mater. Technol. 2020, 5, 1900943.

(11) Kim, Y.; Lim, T.; Kim, C.-H.; Yeo, C. S.; Seo, K.; Kim, S.-M.; Kim, J.; Park, S. Y.; Ju, S.; Yoon, M.-H. Organic Electrochemical Transistor-Based Channel Dimension-Independent Single-Strand Wearable Sweat Sensors. NPG Asia Mater. 2018, 10, 1086-1095.

(12) Wang, Y.; Zhou, Z.; Qing, X.; Zhong, W.; Liu, Q.; Wang, W.; Li, M.; Liu, K.; Wang, D. Ion Sensors Based on Novel Fiber Organic Electrochemical Transistors for Lead Ion Detection. Anal. Bioanal. Chem. 2016, 408, 5779-5787.

(13) Macchia, E.; Romele, P.; Manoli, K.; Ghittorelli, M.; Magliulo, M.; Kovács-Vajna, Z. M.; Torricelli, F.; Torsi, L. Ultra-Sensitive Protein Detection with Organic Electrochemical Transistors Printed on Plastic Substrates. Flexible Printed Electron. 2018, 3, 034002.

(14) Lin, P.; Yan, F.; Yu, J.; Chan, H. L. W.; Yang, M. The Application of Organic Electrochemical Transistors in Cell-based Biosensors. Adv. Mater. 2010, 22, 3655-3660.

(15) Wang, X.; Lu, X.; Liu, B.; Chen, D.; Tong, Y.; Shen, G. Flexible Energy-Storage Devices: Design Consideration and Recent Progress. Adv. Mater. 2014, 26, 4763-4782.

(16) Dubal, D. P. Chapter 7 - Advances in Flexible Supercapacitors for Portable and Wearable Smart Gadgets. Emerging Mater. Energy Convers. Storage 2018, 209-246. 
(17) Palchoudhury, S.; Ramasamy, K.; Gupta, R. K.; Gupta, A. Flexible Supercapacitors: A Materials Perspective. Front. Mater. 2019, $5,83$.

(18) Devillers, N.; Jemei, S.; Péra, M.-C.; Bienaimé, D.; Gustin, F. Review of Characterization Methods for Supercapacitor Modelling. J. Power Sources 2014, 246, 596-608.

(19) Wang, G.; Zhang, L.; Zhang, J. A Review of Electrode Materials for Electrochemical Supercapacitors. Chem. Soc. Rev. 2012, 41, 797828.

(20) González, A.; Goikolea, E.; Barrena, J. A.; Mysyk, R. Review on Supercapacitors: Technologies and Materials. Renewable Sustainable Energy Rev. 2016, 58, 1189-1206.

(21) Sahalianov, I.; Singh, S. K.; Tybrandt, K.; Berggren, M.; Zozoulenko, I. The Intrinsic Volumetric Capacitance of Conducting Polymers: Pseudo-Capacitors or Double-Layer Supercapacitors? RSC Adv. 2019, 9, 42498-42508.

(22) Soavi, F.; Bettini, L. G.; Piseri, P.; Milani, P.; Santoro, C.; Atanassov, P.; Arbizzani, C. Miniaturized Supercapacitors: Key Materials and Structures towards Autonomous and Sustainable Devices and Systems. J. Power Sources 2016, 326, 717-725.

(23) Meng, Q.; Cai, K.; Chen, Y.; Chen, L. Research Progress on Conducting Polymer Based Supercapacitor Electrode Materials. Nano Energy 2017, 36, 268-285.

(24) Zhao, Z.; Richardson, G. F.; Meng, Q.; Zhu, S.; Kuan, H.-C.; Ma, J. PEDOT-Based Composites as Electrode Materials for Supercapacitors. Nanotechnology 2016, 27, 042001.

(25) Kirchmeyer, S.; Reuter, K. Scientific Importance, Properties and Growing Applications of Poly(3,4-Ethylenedioxythiophene). J. Mater. Chem. 2005, 15, 2077-2088.

(26) Snook, G. A.; Peng, C.; Fray, D. J.; Chen, G. Z. Achieving High Electrode Specific Capacitance with Materials of Low Mass Specific Capacitance: Potentiostatically Grown Thick Micro-Nanoporous PEDOT Films. Electrochem. Commun. 2007, 9, 83-88.

(27) Liu, R.; Cho, S. I.; Lee, S. B. Poly (3, 4-Ethylenedioxythiophene) Nanotubes as Electrode Materials for a High-Powered Supercapacitor. Nanotechnology 2008, 19, 215710.

(28) Zhou, H.; Zhi, X. Surfactant-Assisted Potentiodynamically Polymerized PEDOT Fibers for Significantly Improved Electrochemical Capacitive Properties. Mater. Lett. 2018, 221, 309-312.

(29) Pandey, G. P.; Rastogi, A. C.; Westgate, C. R. All-Solid-State Supercapacitors with Poly (3, 4-Ethylenedioxythiophene)-Coated Carbon Fiber Paper Electrodes and Ionic Liquid Gel Polymer Electrolyte. J. Power Sources 2014, 245, 857-865.

(30) Anothumakkool, B.; Soni, R.; Bhange, S. N.; Kurungot, S. Novel Scalable Synthesis of Highly Conducting and Robust PEDOT Paper for a High Performance Flexible Solid Supercapacitor. Energy Environ. Sci. 2015, 8, 1339-1347.

(31) D’Arcy, J. M.; El-Kady, M. F.; Khine, P. P.; Zhang, L.; Lee, S. H.; Davis, N. R.; Liu, D. S.; Yeung, M. T.; Kim, S. Y.; Turner, C. L.; Lech, A. T.; Hammond, P. T.; Kaner, R. B. Vapor-Phase Polymerization of Nanofibrillar Poly(3,4-Ethylenedioxythiophene) for Supercapacitors. ACS Nano 2014, 8, 1500-1510.

(32) Rajesh, M.; Raj, C. J.; Manikandan, R.; Kim, B. C.; Park, S. Y.; Yu, K. H. A High Performance PEDOT/PEDOT Symmetric Supercapacitor by Facile in-Situ Hydrothermal Polymerization of PEDOT Nanostructures on Flexible Carbon Fibre Cloth Electrodes. Mater. Today Energy 2017, 6, 96-104.

(33) Edberg, J.; Inganäs, O.; Engquist, I.; Berggren, M. Boosting the Capacity of All-Organic Paper Supercapacitors Using Wood Derivatives. J. Mater. Chem. A 2018, 6, 145-152.

(34) Yang, Z.; Ma, J.; Bai, B.; Qiu, A.; Losic, D.; Shi, D.; Chen, M. Free-Standing PEDOT/Polyaniline Conductive Polymer Hydrogel for Flexible Solid-State Supercapacitors. Electrochim. Acta 2019, 322, 134769.

(35) Alvi, F.; Ram, M. K.; Basnayaka, P. A.; Stefanakos, E.; Goswami, Y.; Kumar, A. Graphene-Polyethylenedioxythiophene Conducting Polymer Nanocomposite Based Supercapacitor. Electrochim. Acta 2011, 56, 9406-9412.
(36) Li, K.-B.; Shi, D.-W.; Cai, Z.-Y.; Zhang, G.-L.; Huang, Q.-A.; Liu, D.; Yang, C.-P. Studies on the Equivalent Serial Resistance of Carbon Supercapacitor. Electrochim. Acta 2015, 174, 596-600.

(37) Ranjusha, R.; Sajesh, K. M.; Roshny, S.; Lakshmi, V.; Anjali, P.; Sonia, T. S.; Sreekumaran Nair, A.; Subramanian, K. R. V.; Nair, S. V.; Chennazhi, K. P.; Balakrishnan, A. Supercapacitors Based on Freeze Dried $\mathrm{MnO} 2$ Embedded PEDOT: PSS Hybrid Sponges. Microporous Mesoporous Mater. 2014, 186, 30-36.

(38) Liu, C.; Jiang, F.; Huang, M.; Lu, B.; Yue, R.; Xu, J. FreeStanding PEDOT-PSS $/ \mathrm{Ca}_{3} \mathrm{Co}_{4} \mathrm{O}_{9}$ Composite Films as Novel Thermoelectric Materials. J. Electron. Mater. 2011, 40, 948-952.

(39) Ni, D.; Chen, Y.; Song, H.; Liu, C.; Yang, X.; Cai, K. FreeStanding and Highly Conductive PEDOT Nanowire Films for HighPerformance All-Solid-State Supercapacitors. J. Mater. Chem. A 2019, 7, 1323-1333.

(40) Jayaram, A. K.; Pitsalidis, C.; Tan, E.; Moysidou, C.-M.; De Volder, M. F. L.; Kim, J.-S.; Owens, R. M. 3D Hybrid Scaffolds Based on PEDOT:PSS/MWCNT Composites. Front. Chem. 2019, 363.

(41) Zhao, Q.; Wang, G.; Yan, K.; Yan, J.; Wang, J. Binder-Free Porous PEDOT Electrodes for Flexible Supercapacitors. J. Appl. Polym. Sci. 2015, 132 (), DOI: 10.1002/app.42549.

(42) Cheng, T.; Zhang, Y.-Z.; Zhang, J.-D.; Lai, W.-Y.; Huang, W. High-Performance Free-Standing PEDOT:PSS Electrodes for Flexible and Transparent All-Solid-State Supercapacitors. J. Mater. Chem. A 2016, 4, 10493-10499.

(43) Diao, Y.; Chen, H.; Lu, Y.; Santino, L. M.; Wang, H.; D’Arcy, J. M. Converting Rust to PEDOT Nanofibers for Supercapacitors. ACS Appl. Energy Mater. 2019, 2, 3435-3444.

(44) Donahue, M. J.; Sanchez-Sanchez, A.; Inal, S.; Qu, J.; Owens, R. M.; Mecerreyes, D.; Malliaras, G. G.; Martin, D. C. Tailoring PEDOT Properties for Applications in Bioelectronics. Mater. Sci. Eng. R 2020, 140,100546

(45) Elschner, A.; Kirchmeyer, S.; Lovenich, W.; Merker, U.; Reuter, K. PEDOT: Principles and Applications of an Intrinsically Conductive Polymer; CRC press: 2010, DOI: 10.1201/b10318.

(46) Gomez, N.; Lee, J. Y.; Nickels, J. D.; Schmidt, C. E. Micropatterned Polypyrrole: A Combination of Electrical and Topographical Characteristics for the Stimulation of Cells. Adv. Funct. Mater. 2007, 17, 1645-1653.

(47) Yang, J.; Martin, D. C. Impedance Spectroscopy and Nanoindentation of Conducting Poly (3, 4-Ethylenedioxythiophene) Coatings on Microfabricated Neural Prosthetic Devices. J. Mater. Res. 2006, 21, 1124-1132.

(48) Wang, X.; Egan, C. E.; Zhou, M.; Prince, K.; Mitchell, D. R. G.; Caruso, R. A. Effective Gel for Gold Nanoparticle Formation, Support and Metal Oxide Templating. Chem. Commun. 2007, 3060-3062.

(49) Koo, H.-J.; Chang, S. T.; Slocik, J. M.; Naik, R. R.; Velev, O. D. Aqueous Soft Matter Based Photovoltaic Devices. J. Mater. Chem. 2011, 21, 72-79.

(50) Zhou, Y.; Jin, P.; Zhou, Y.; Zhu, Y. High-Performance Symmetric Supercapacitors Based on Carbon Nanotube/Graphite Nanofiber Nanocomposites. Sci. Rep. 2018, 8, 9005.

(51) Yan, J.; Ren, C. E.; Maleski, K.; Hatter, C. B.; Anasori, B.; Urbankowski, P.; Sarycheva, A.; Gogotsi, Y. Flexible MXene/ Graphene Films for Ultrafast Supercapacitors with Outstanding Volumetric Capacitance. Adv. Funct. Mater. 2017, 27, 1701264.

(52) Wang, H.; Yi, H.; Chen, X.; Wang, X. Asymmetric Supercapacitors Based on Nano-Architectured Nickel Oxide/ Graphene Foam and Hierarchical Porous Nitrogen-Doped Carbon Nanotubes with Ultrahigh-Rate Performance. J. Mater. Chem. A 2014, $2,3223-3230$.

(53) Salunkhe, R. R.; Tang, J.; Kobayashi, N.; Kim, J.; Ide, Y.; Tominaka, S.; Kim, J. H.; Yamauchi, Y. Ultrahigh Performance Supercapacitors Utilizing Core-Shell Nanoarchitectures from a Metal-Organic Framework-Derived Nanoporous Carbon and a Conducting Polymer. Chem. Sci. 2016, 7, 5704-5713.

(54) Wustoni, S.; Combe, C.; Ohayon, D.; Akhtar, M. H.; McCulloch, I.; Inal, S. Membrane-Free Detection of Metal Cations 
with an Organic Electrochemical Transistor. Adv. Funct. Mater. 2019, 29, 1904403.

(55) Mantione, D.; Del Agua, I.; Sanchez-Sanchez, A.; Mecerreyes, D. Poly (3, 4-Ethylenedioxythiophene)(PEDOT) Derivatives: Innovative Conductive Polymers for Bioelectronics. Polymers 2017, 9, 354.

(56) Li, C.; Bai, H.; Shi, G. Conducting Polymer Nanomaterials: Electrosynthesis and Applications. Chem. Soc. Rev. 2009, 38, 23972409.

(57) Brillas, E.; Oliver, R.; Estrany, F.; Rodríguez, E.; Tejero, S. Anodic Polymerization of $\alpha$-Tetrathiophene in Organic Medium. Doping with Perchlorate Ion and Properties of Conducting Polymer. Electrochim. Acta 2002, 47, 1623-1631.

(58) Arias-Pardilla, J.; Giménez-Gómez, P. A.; de la Peña, A.; Segura, J. L.; Otero, T. F. Synthesis, Electropolymerization and Characterization of a Cross-Linked PEDOT Derivative. J. Mater. Chem. 2012, 22, 4944-4952.

(59) Aradilla, D.; Estrany, F.; Alemán, C. Symmetric Supercapacitors Based on Multilayers of Conducting Polymers. J. Phys. Chem. C 2011, 115, 8430-8438.

(60) Li, Y.; Hong, N. An Efficient Hole Transport Material Based on PEDOT Dispersed with Lignosulfonate: Preparation, Characterization and Performance in Polymer Solar Cells. J. Mater. Chem. A 2015, 3, 21537-21544.

(61) Zhao, Q.; Jamal, R.; Zhang, L.; Wang, M.; Abdiryim, T. The Structure and Properties of PEDOT Synthesized by Template-Free Solution Method. Nanoscale Res. Lett. 2014, 9, 557.

(62) Ghosh, S.; Remita, H.; Ramos, L.; Dazzi, A.; Deniset-Besseau, A.; Beaunier, P.; Goubard, F.; Aubert, P.-H.; Brisset, F.; Remita, S. PEDOT Nanostructures Synthesized in Hexagonal Mesophases. New J. Chem. 2014, 38, 1106-1115.

(63) Cho, M. S.; Kim, S. Y.; Nam, J. D.; Lee, Y. Preparation of PEDOT/Cu Composite Film by in Situ Redox Reaction between EDOT and Copper(II) Chloride. Synth. Met. 2008, 158, 865-869.

(64) Hohnholz, D.; MacDiarmid, A. G.; Sarno, D. M.; Jones, W. E., Jr. Uniform Thin Films of Poly-3,4-Ethylenedioxythiophene (PEDOT) Prepared by in-Situ Deposition. Chem. Commun. 2001, 2444-2445.

(65) Österholm, A. M.; Ponder, J. F., Jr.; Kerszulis, J. A.; Reynolds, J. R. Solution Processed PEDOT Analogues in Electrochemical Supercapacitors. ACS Appl. Mater. Interfaces 2016, 8, 13492-13498.

(66) Zhang, M.; Zhou, Q.; Chen, J.; Yu, X.; Huang, L.; Li, Y.; Li, C.; Shi, G. An Ultrahigh-Rate Electrochemical Capacitor Based on Solution-Processed Highly Conductive PEDOT:PSS Films for AC Line-Filtering. Energy Environ. Sci. 2016, 9, 2005-2010.

(67) Sharma, P.; Bhatti, T. S. A Review on Electrochemical DoubleLayer Capacitors. Energy Convers. Manage. 2010, 51, 2901-2912.

(68) Mondal, S.; Rana, U.; Malik, S. Reduced Graphene Oxide/ Fe3O4/Polyaniline Nanostructures as Electrode Materials for an AllSolid-State Hybrid Supercapacitor. J. Phys. Chem. C 2017, 121, 75737583.

(69) Mehta, G. K.; Kondaveeti, S.; Siddhanta, A. K. Facile Synthesis of Agarose-l-Phenylalanine Ester Hydrogels. Polym. Chem. 2011, 2, 2334-2340.

(70) Pervez, S.; Nawaz, M. A.; Aman, A.; Qayyum, S.; Nawaz, F.; Qader, S. A. U. Agarose Hydrogel Beads: An Effective Approach to Improve the Catalytic Activity, Stability and Reusability of Fungal Amyloglucosidase of GH15 Family. Catal. Lett. 2018, 148, 26432653.

(71) Clar, J. G.; Silvera Batista, C. A.; Youn, S.; Bonzongo, J.-C. J.; Ziegler, K. J. Interactive Forces between Sodium Dodecyl SulfateSuspended Single-Walled Carbon Nanotubes and Agarose Gels. J. Am. Chem. Soc. 2013, 135, 17758-17767.

(72) Raphael, E.; Avellaneda, C. O.; Manzolli, B.; Pawlicka, A. AgarBased Films for Application as Polymer Electrolytes. Electrochim. Acta 2010, 55, 1455-1459.

(73) Trivedi, T. J.; Bhattacharjya, D.; Yu, J.-S.; Kumar, A. Functionalized Agarose Self-Healing Ionogels Suitable for Supercapacitors. ChemSusChem 2015, 8, 3294-3303.
(74) Wang, Z.; Chu, X.; Xu, Z.; Su, H.; Yan, C.; Liu, F.; Gu, B.; Huang, H.; Xiong, D.; Zhang, H.; Deng, W.; Zhang, H.; Yang, W. Extremely Low Self-Discharge Solid-State Supercapacitors via the Confinement Effect of Ion Transfer. J. Mater. Chem. A 2019, 7, 86338640.

(75) Conway, B. E.; Pell, W. G.; Liu, T.-C. Diagnostic Analyses for Mechanisms of Self-Discharge of Electrochemical Capacitors and Batteries. J. Power Sources 1997, 65, 53-59.

(76) Davis, M. A.; Andreas, H. A. Identification and Isolation of Carbon Oxidation and Charge Redistribution as Self-Discharge Mechanisms in Reduced Graphene Oxide Electrochemical Capacitor Electrodes. Carbon 2018, 139, 299-308.

(77) Conway, B. E. Electrochemical Supercapacitors: Scientific Fundamentals and Technological Applications; Springer Science \& Business Media: 2013.

(78) Skotheim, T. A.; Reynolds, J. Conjugated Polymers: Theory, Synthesis, Properties, and Characterization; CRC press: 2006, DOI: $10.1201 / 9781420043594$.

(79) Du, F.-P.; Cao, N.-N.; Zhang, Y.-F.; Fu, P.; Wu, Y.-G.; Lin, Z.D.; Shi, R.; Amini, A.; Cheng, C. PEDOT:PSS/Graphene Quantum Dots Films with Enhanced Thermoelectric Properties via Strong Interfacial Interaction and Phase Separation. Sci. Rep. 2018, 8, 6441.

(80) Barsch, U.; Beck, F. Anodic Overoxidation of Polythiophenes in Wet Acetonitrile Electrolytes. Electrochim. Acta 1996, 41, 1761-1771.

(81) Li, Y.; Sun, J.; Wang, J.; Bian, C.; Tong, J.; Li, Y.; Xia, S. A Single-Layer Structured Microbial Sensor for Fast Detection of Biochemical Oxygen Demand. Biochem. Eng. J. 2016, 112, 219-225.

(82) Xu, Y.; Lin, Z.; Huang, X.; Liu, Y.; Huang, Y.; Duan, X. Flexible Solid-State Supercapacitors Based on Three-Dimensional Graphene Hydrogel Films. ACS Nano 2013, 7, 4042-4049.

(83) Tang, P.; Zhao, Y.; Xu, C. Step-by-Step Assembled Poly (3, 4Ethylenedioxythiophene)/Manganese Dioxide Composite Electrodes: Tuning the Structure for High Electrochemical Performance. Electrochim. Acta 2013, 89, 300-309.

(84) He, X.; Yang, W.; Mao, X.; Xu, L.; Zhou, Y.; Chen, Y.; Zhao, Y.; Yang, Y.; Xu, J. All-Solid State Symmetric Supercapacitors Based on Compressible and Flexible Free-Standing 3D Carbon Nanotubes (CNTs)/Poly (3, 4-Ethylenedioxythiophene)(PEDOT) Sponge Electrodes. J. Power Sources 2018, 376, 138-146.

(85) Ahmed, S.; Rafat, M. Hydrothermal Synthesis of PEDOT/ RGO Composite for Supercapacitor Applications. Mater. Res. Express 2018, 5, 015507. 